Board of Governors of the Federal Reserve System

International Finance Discussion Papers

Number 635

April 1999

\title{
EXACT UTILITIES UNDER ALTERNATIVE MONETARY RULES IN A SIMPLE MACRO MODEL WITH OPTIMIZING AGENTS
}

Dale W. Henderson and Jinill Kim

NOTE: International Finance Discussion Papers are preliminary materials circulated to stimulate discussion and critical comment. References in publications to International Finance Discussion Papers (other than an acknowledgment that the writer has had access to unpublished material) should be cleared with the author or authors. Recent IFDPs are available on the Web at www.bog.frb.fed.us. 


\title{
EXACT UTILITIES UNDER ALTERNATIVE MONETARY RULES IN A SIMPLE MACRO MODEL WITH OPTIMIZING AGENTS
}

\author{
Dale W. Henderson and Jinill Kim*
}

\begin{abstract}
We construct an optimizing-agent model of a closed economy which is simple enough that we can use it to make exact utility calculations. There is a stabilization problem because there are one-period nominal contracts for wages, or prices, or both and shocks that are unknown at the time when contracts are signed. We evaluate alternative monetary policy rules using the utility function of the representative agent. Fully optimal policy can attain the Pareto-optimal equilibrium. Fully optimal policy is contrasted with both 'naive' and 'sophisticated' simple rules that involve, respectively, complete stabilization and optimal stabilization of one variable or a combination two variables. With wage contracts, outcomes depend crucially on whether there are also price contracts. For example, if labor supply is relatively inelastic, for productivity shocks, nominal income stabilization yields higher welfare when there are no price contracts. However, with price contracts, outcomes are independent of whether there are wage contracts, except, of course, for nominal wage outcomes.
\end{abstract}

Keywords: monetary policy, stabilization, sticky wages, sticky prices, wage contracts, price contracts.

"This paper was prepared for the "Conference in Celebration of the Contributions of Robert Flood" held at the International Monetary Fund on January 15-16, 1999. It will appear as both Henderson and Kim (1999a) and Henderson and Kim (1999b). We would like to thank Jo Anna Gray, our discussant, for helpful comments and Charles Engel for suggesting that we change our specification of the objective function of firms to the current one. The views in this paper are solely the responsibility of the authors and should not be interpreted as reflecting the views of the Board of Governors of the Federal Reserve System or any other person associated with the Federal Reserve System. The email addresses of the authors are hendersd@frb.gov and jk9n@virginia.edu respectively. 


\section{Introduction}

Interest in improving the analytical foundations of monetary stabilization policy is at a cyclical peak. This paper is a contribution to that endeavor. We construct an optimizing-agent model of a closed economy which is simple enough that we can make exact utility calculations. In this model, there is a stabilization problem because there are one-period nominal contracts for wages, or prices, or both and shocks that are unknown at the time when contracts are signed. We evaluate alternative monetary policy rules using as a criterion the utility function of the representative agent.

One well known advantage of using exact utility calculations is that it makes it possible to analyze shocks with large as well as small variances. An unexpected advantage is that it actually simplifies the algebraic derivations in our model. However, when shocks have small variances, it yields no advantage for welfare analysis in our model; welfare rankings are the same with exact and approximate utility calculations. ${ }^{1}$

We focus on two cases, (1) wage contracts and flexible prices and (2) wage and price contracts. If wages are fixed by contracts, for some shocks the attractiveness of some simple rules depends crucially on whether prices are also fixed by contracts. We can limit our focus to two cases because, as we show, the outcomes in the third case, price contracts and flexible wages, are the same as the outcomes in the case of wage and price contracts for all variables except, of course, for the nominal wage. ${ }^{2}$

We calculate the fully optimal rule under complete information for each of our two cases of interest. This rule can attain the Pareto-optimal equilibrium because we assume one-period nominal contracts, so the policymaker does not face a tradeoff. ${ }^{3}$ Then we contrast the performance of the fully optimal policy with both 'naive' and 'sophisticated' versions of some simple rules. Naive simple rules involve complete stabilization of one variable or a combination of two variables. Sophisticated simple rules involve optimal stabilization of one variable or a combination of two variables. We consider sophisticated versions of simple rules in an attempt to put these rules in the best possible light.

Our paper is closely related to two sets of recent studies. The studies in one set contain evaluations of alternative monetary policies using approximate solutions of models with optimizing-agents. ${ }^{4}$ Of course, the authors of these studies have used

\footnotetext{
${ }^{1}$ This assertion can be confirmed using the methods developed in Rotemberg and Woodford (1998) and imposing our assumption that subsidies are used to eliminate the output and employement distortions arising from monopolistic competition. Even when the variances of shocks are small, aproximate solutions yield incorrect welfare rankings in some models. For example, Kim and Kim (1999) show that in a model of international risk sharing a standard approximation implies that welfare is lower with a complete market than with autarky.

${ }^{2}$ However, if prices are fixed by staggered contracts instead of by one-period contracts (or by synchronized multiperiod contracts), results depend crucially on whether wages are fixed by contracts or are flexible as shown by Erceg, Henderson, and Levin (1999).

${ }^{3}$ Even the fully optimal policy under complete information cannot attain the Pareto-optimal equilibrium if both wages and prices are fixed by staggered contracts as shown by Erceg, Henderson, and Levin (1999).

${ }^{4}$ This set includes Ireland (1997), Goodfriend and King (1997), Rotemberg and Woodford (1998), Henderson and Kim (1999a), and the King and Wolman and Rotemberg and Woodford papers in Taylor (1999).
} 
approximate solutions because their models are complex enough that obtaining exact solutions would be relatively difficult and costly if it were even feasible. It seems useful to supplement their analysis with analysis of models that are simple enough that obtaining exact solutions is relatively easy.

The studies in the other set are based on two-country models in which exact utility calculations are possible. ${ }^{5}$ Our emphasis differs from the emphasis in these studies. We focus on the welfare effects of alternative monetary stabilization rules in a stochastic model. In contrast, the other studies focus either on the welfare effects of a one-time increase in the money supply in a perfect foresight model, on the implications of alternative money supply processes for asset returns in a stochastic model, or on a welfare comparison of fixed and flexible exchange rates in a stochastic model. Another notable difference between our paper and the other studies is that for us the interest rate, not the money supply, is the instrument of monetary policy.

The rest of this paper is organized into five more sections. Section 2 is a description of our model. We devote section 3 to the benchmark version with flexible wages and prices. In sections 4 and 5, we analyze alternative monetary policy rules in versions with wage contracts and flexible prices and with both wage and price contracts, respectively. Section 6 contains our conclusions. The demonstration that the version with price contracts and flexible wages yields the same outcomes as the version with both wage and price contracts (except for nominal wages) is in the Appendix.

\section{The Model}

In this section we describe our model. We discuss the behavior of firms, households, and the government in successive subsections.

\section{$2.1 \quad$ Firms}

A continuum of 'identical' monopolistically competitive firms is distributed on the unit interval, $f \in[0,1]$. With no price contracts, firms set their prices for period $t$ based on period $t$ information. With one-period price contracts, firms set prices for period $t+1$ based on period $t$ information and agree to supply whatever their customers demand at those prices. In either case, the problem of firm $f$ in period $t$ is to find the

$$
\max _{\left\{P_{f, t+j}\right\}} \mathcal{E}_{t} \tilde{\delta}_{t, t+j}\left(s_{P} \mathrm{P}_{f, t+j} \mathrm{Y}_{f, t+j}-W_{t+j} L_{f, t+j}\right)
$$

where capital letters without serifs represent choice variables of individual firms or households and capital letters with serifs represent indexes that include all firms or households. The subscript $j$ takes on the value 0 if there are no price contracts and the value 1 if there are price contracts. In period $t+j$, firm $f$ sets the price

\footnotetext{
${ }^{5}$ This set includes Corsetti and Pesenti (1998), which is based on a perfect foresight model, and Obstfeld and Rogoff (1998) Devereux and Engel (1998), and Engel (1999a), and Engel (1999b) which are based on stochastic models.
} 
$\mathrm{P}_{f, t+j}$, produces output $\mathrm{Y}_{f, t+j}$, and employs the amount $L_{f, t+j}$ of a labor index $L_{t+j}$ for which it pays the wage index $W_{t+j}$ per unit:

$$
L_{t+j}=\int_{0}^{1} L_{f, t+j} d f=\left(\int_{0}^{1} \mathrm{~L}_{h, t+j}^{\frac{1}{\theta_{W}}} d h\right)^{\theta_{W}} \quad W_{t+j}=\left(\int_{0}^{1} \mathrm{~W}_{h, t+j}^{\frac{1}{1-\theta_{W}}} d h\right)^{1-\theta_{W}}
$$

where $\mathrm{L}_{h, t+j}$ is the amount of labor supplied by household $h$ in period $t+j, \mathrm{~W}_{h, t+j}$ is the wage charged by household $h$ in period $t+j$, and $\theta_{W}>1$. Firm $f$ choose quantities of $\mathrm{L}_{h, t+j}$ to minimize the cost of producing a unit of $L_{f, t+j}$ given the $\mathrm{W}_{h, t+j}$, and $W_{t+j}$ is the minimum cost. All firms receive an ad valorem output subsidy, $s_{P}$. Each element of the infinite dimensional vector $\tilde{\delta}_{t, t+j}$ is the price of a claim to one dollar delivered in a particular state in period $t+1$ divided by the probability of that state. We use $\mathcal{E}_{t}$ to indicate an expectation taken over the states in period $t+j$ based on period $t$ information. The production function of firm $f$ is $^{6}$

$$
\mathrm{Y}_{f, t+j}=\frac{L_{f, t+j}^{(1-\alpha)} X_{t+j}}{1-\alpha}
$$

where $X_{t+j}$ is a productivity shock that hits all firms, and $x_{t+j}=\ln X_{t+j} \backsim N\left(0,2 \sigma_{x}^{2}\right)$. An expression for $L_{f, t+j}$ is obtained by inverting this production function.

Relative demand for output of firm $f$ is a decreasing function of its relative price:

$$
\frac{\mathrm{Y}_{f, t+j}}{Y_{t+j}}=\left(\frac{\mathrm{P}_{f, t+j}}{P_{t+j}}\right)^{-\frac{\theta_{P}}{\theta_{P}-1}}
$$

where $\theta_{P}>1$. In equation (4), $Y_{t+j}$ is an index made up of the output of all firms and $P_{t+j}$ is a price index which is the price of a unit of the output index:

$$
Y_{t+j}=\int_{0}^{1} Y_{h, t+j} d h=\left(\int_{0}^{1} \mathrm{Y}_{f, t+j}^{\frac{1}{\theta_{p}}}\right)^{\theta_{P}} \quad P_{t+j}=\left(\int_{0}^{1} \mathrm{P}_{f, t+j}^{\frac{1}{1-\theta_{P}}} d f\right)^{1-\theta_{P}}
$$

where $Y_{h, t+j}$ is the amount of the output index purchased by household $h$ in period $t+j$. Household $h$ chooses quantities of $\mathrm{Y}_{f, t+j}$ to minimize the cost of producing a unit of $Y_{h, t+j}$ given the $\mathrm{P}_{f, t+j}$, and $P_{t+j}$ is the minimum cost.

To maximize profits, a firm must set its price so that expected discounted marginal revenue equals expected discounted marginal cost:

$$
s_{P}\left(\frac{\theta_{P}}{\theta_{P}-1}-1\right) \mathcal{E}_{t}\left(\tilde{\delta}_{t, t+j} Y_{f, t+j}\right)=\left(\frac{\theta_{P}}{\theta_{P}-1}\right) \mathcal{E}_{t}\left(\frac{\tilde{\delta}_{t, t+j} W_{t+j} L_{f, t+j}^{\alpha} Y_{f, t+j}}{P_{f, t+j} X_{t+j}}\right)
$$

\footnotetext{
${ }^{6}$ That is, we assume for simplicity that there are no factors of production other than labour and no fixed costs. Kim (1998) shows that our formulation can be viewed as a model with capital in which the marginal adjustment cost for the first unit of net investment approaches infinity. Kim (1997) explores the implications of allowing for fixed costs.
} 
Since firms are identical,

$$
L_{f,+j}=L_{+j} \quad Y_{f,+j}=Y_{+j} \quad P_{f,+j}=P_{+j}
$$

where we omit $t$ subscripts in the rest of this subsection for simplicity. Therefore, the equalities in (7) imply that the 'aggregate production function' and 'aggregate price equation' are, respectively,

$$
\begin{gathered}
Y_{+j}=\frac{L_{+j}^{(1-\alpha)} X_{+j}}{1-\alpha} \\
s_{P} \mathcal{E}\left(\tilde{\delta}_{+j} Y_{+j}\right)=\theta_{P} \mathcal{E}\left(\frac{\tilde{\delta}_{+j} W_{+j} L_{+j}^{\alpha} Y_{+j}}{P_{+j} X_{+j}}\right)
\end{gathered}
$$

When $j=0$ so that period $t$ prices are set on the basis of period $t$ information, the aggregate price equation (9) can be rewritten as

$$
\left(\frac{s_{P}}{\theta_{P}}\right) \frac{X}{L^{\alpha}}=\frac{W}{P}
$$

which states that $P$ must be chosen so that the marginal value product of labor (the gross subsidy rate over the markup parameter times the marginal product of labor) equals the real wage. We assume that the government sets $s_{P}=\theta_{P}$ to offset the effect of the distortion associated with monopolistic competition in the goods market. Under this assumption, the ratio $\frac{s_{P}}{\theta_{P}}$ equals one, so it does not appear in what follows, and the implied version of equation (10) states that the marginal product of labor must equal the real wage.

\subsection{Households}

A continuum of 'identical' households is distributed on the unit interval, $h \in[0,1]$. With no wage contracts, households set their wages for period $t$ based on period $t$ information, but with wage contracts they set their wages for period $t+1$ based on period $t$ information. The problem of household $h$ in period $t$ is to find the

$$
\max _{\left\{C_{h, s}, M_{h, s}, B_{h, s}, B_{h, s}^{g}, W_{h, s+j}\right\}} \mathcal{E}_{t} \sum_{s=t}^{\infty} \beta^{s-t}\left(\frac{\mathcal{C}_{h, s}^{1-\rho}-1}{1-\rho}-\frac{\chi_{0} \mathrm{~L}_{h, s}^{1+\chi}}{Z_{s}(1+\chi)}\right) U_{s}
$$

subject to

$$
\begin{aligned}
C_{h, s}= & \frac{s_{W} \mathrm{~W}_{h, s} \mathrm{~L}_{h, s}}{P_{s}}+\frac{\Gamma_{s}}{P_{s}}-T_{h, s} \\
& -\frac{M_{h, s}-M_{h, s-1}+\delta_{s, s+1} B_{h, s}-B_{h, s-1}+B_{h, s}^{g}-I_{-1} B_{h, s-1}^{g}}{P_{s}}
\end{aligned}
$$




$$
\begin{gathered}
\mathcal{C}_{h, s}=\min \left(C_{h, s}, \frac{M_{h, s}}{P_{s} V_{s}}\right), \quad M_{h, s}=P_{s} C_{h, s} V_{s} \quad \mathcal{C}_{h, s}=C_{h, s} \\
\frac{\mathrm{L}_{h, s}}{L_{s}}=\left(\frac{\mathrm{W}_{h, s}}{W_{s}}\right)^{-\frac{\theta_{W}}{\theta_{W}-1}}
\end{gathered}
$$

In period $s$, household $h$ chooses its expenditure on the output index $\left(C_{h, s}=Y_{h, s}\right)$ and its holdings of money, $M_{h, s}$, which imply a consumption realization, $\mathcal{C}_{h, s}$ Household $h$ also chooses its wage rate in period $s+j, \mathrm{~W}_{h, s+j}$, and agrees to supply however many units of its labor, $\mathrm{L}_{h, s+j}$, firms want at this wage where the subscript $j$ takes on the value 0 if there are no wage contracts and the value 1 if there are wage contracts. In addition, in period $s$, household $h$ chooses its holdings of claims to a unit of currency in the various states in period $s+1$. Each element in the infinite-dimensional vector $\delta_{s, s+1}$ represents the price of an asset that will pay one unit of currency in a particular state of nature in the subsequent period, while the corresponding element of the vector $B_{h, s}$ represents the quantity of such clams purchased by the household. The scalar variable $B_{h, s-1}$ represents the value of the households's claims given the current state of nature. Household $h$ also chooses its holding of government bonds $B_{h, s}^{g}$, which pay $I_{s}$ units of currency in every state of nature in period $s+1$. Household $h$ receives an aliquot share, $\Gamma_{s}$, of aggregate profits and pays lump sum taxes, $T_{h, s}{ }^{7}$ All households receive an ad valorem labor subsidy, $s_{W}$. There are goods demand, $U_{s}$, money demand, $V_{s}$, and labor supply shocks, $Z_{s}$, that hit all consumers. We assume that the shocks $U_{s}, X_{s}$, and $Z_{s}$ have lognormal distributions. ${ }^{8}$. We impose the restrictions that $0<\beta<1, \rho \geq 1$, and $\chi \geq 0 .{ }^{9} \quad \mathcal{E}_{t}$ indicates an expectation over the various states in period $s$ based on period $t$ information.

According to equation (11), period utility depends positively on the consumption realization and negatively on labor supply. The period budget constraint, equation (12), states that consumption expenditure must equal disposable income minus asset accumulation. According to the first equality in equation (13), the consumption realization is equal to the minimum of consumption expenditure and adjusted real balances (real balances divided by a money demand shock). We assume that household $h$ always keeps consumption expenditure and adjusted real balances equal to one another (the second equality in equation (13)) so that the consumption realization is always equal to consumption expenditure (the third equality in equation (13)). Each household is a monopolistically competitive supplier of its unique labor input. Rel-

${ }^{7}$ These equal shares exhaust aggregate profits:

$$
\int_{0}^{1} \Gamma_{s} d h=\int_{0}^{1}\left(s_{P} \mathrm{P}_{f, s} \mathrm{Y}_{f, s}-W_{s} L_{f, s}\right) d f
$$

${ }^{8}$ That is, we assume that $u_{s}=\log U_{s} \backsim N\left(0,2 \sigma_{u}^{2}\right), v_{s}=\log V_{s} \backsim N\left(0,2 \sigma_{v}^{2}\right)$, and $z_{s}=\log Z_{s} \backsim$ $N\left(0,2 \sigma_{z}^{2}\right)$.

${ }^{9}$ As is well known, given the form that we have assumed for the utility of consumption, as $\rho \rightarrow 1$, the utility of consumption approaches $c=\ln C$. 
ative demand for labor of household $h$ is a decreasing function of its relative wage as shown in equation (14).

Substituting equation (13) into equation (11), substituting equation (14) into equation (12), constructing a Lagrangian expression with the multiplier $\eta_{h, s}$ associated with the period budget constraint for period $s$, and differentiating yields the first order conditions for household $h$ for consumption, contingent claims, and government bonds for period $t$ and for the nominal wage in period $t+j, j=0$ or 1 :

$$
\begin{gathered}
\frac{U_{t}}{C_{h, t}^{\rho}}=\eta_{h, t} \\
\frac{\delta_{t, t+1} \eta_{h, t}}{P_{t}}=\frac{\beta \eta_{h, t+1}}{P_{t+1}} \\
\frac{\eta_{h, t}}{P_{t}}=\beta I_{t} \mathcal{E}_{t}\left(\frac{\eta_{h, t+1}}{P_{t+1}}\right) \\
\chi_{0}\left(\frac{\theta_{W}}{\theta_{W}-1}\right) \mathcal{E}_{t}\left(\frac{\left(\mathrm{L}_{h, t+j}\right)^{\chi} \mathrm{L}_{h, t+j} U_{t+j}}{W_{h, t+j} Z_{t}}\right)=s_{W}\left(\frac{\theta_{W}}{\theta_{W}-1}-1\right) \mathcal{E}_{t}\left(\frac{\eta_{h, t+j} \mathrm{~L}_{h, t+j}}{P_{t+j}}\right) \\
M_{h, t}=P_{t} C_{h, t} V_{t}
\end{gathered}
$$

where the condition that consumption must equal adjusted real balances is repeated for convenience. The gross nominal interest rate, $I_{t}$, one plus the nominal interest rate, $i_{t}$, must be equal to one over the cost of acquiring claims to one unit of currency in every state of nature in period $t+1$ :

$$
I_{t}=1+i_{t}=\frac{1}{\int \delta_{t, t+1}}
$$

where the integral is over the states of nature in period $t+1$. Hereafter, we refer to the gross nominal interest rate as the interest rate and omit all $t$ subscripts.

These first order conditions have implications for relationships among aggregate variables. Since households are identical,

$$
C_{h}=C, \mathrm{~L}_{h}=L, W_{h}=W, T_{h}=T, M_{h}=M, B_{h}=B, \eta_{h}=\eta
$$

Eliminating $\eta$ and $\eta_{+1}$ using the condition that in each period in each state

$$
\frac{U_{+j}}{C_{+j}^{\rho}}=\eta_{+j}
$$


yields the 'aggregate first-order conditions for the state contingent contracts,' the 'aggregate consumption Euler equation,' the 'aggregate wage equation,' and the money market equilibrium condition:

$$
\begin{aligned}
& \delta_{t, t+1}\left(\frac{U}{P C^{\rho}}\right)=\beta\left(\frac{U_{+1}}{P_{+1} C_{+1}^{\rho}}\right) \\
& \frac{U}{P C^{\rho}}=\beta I \mathcal{E}\left(\frac{U_{+1}}{P_{+1} C_{+1}^{\rho}}\right) \\
& \theta_{W} \chi_{0} \mathcal{E}\left(\frac{L_{+j}^{1+\chi} U_{+j}}{W_{+j} Z_{+j}}\right)=s_{W} \mathcal{E}\left(\frac{L_{+j} U_{+j}}{P_{+j} C_{+j}^{\rho}}\right) \\
& M=P C V
\end{aligned}
$$

When $j=0$ so that consumers act on the basis of current information, conditions (24) and (25) can be rewritten as

$$
\begin{gathered}
\frac{U}{P C^{\rho}}=\beta I \mathcal{E}\left(\frac{U_{+1}}{P_{+1} C_{+1}^{\rho}}\right) \\
\left(\frac{s_{W}}{\theta_{W}}\right) \frac{W}{P}=\frac{\chi_{0} L^{\chi} C^{\rho}}{Z}
\end{gathered}
$$

Equation (27) states that $W$ must be chosen so that the utility forgone by not spending the marginal dollar on consumption today equals the discounted expected utility of investing that dollar in a riskless security and spending it on consumption tomorrow. Equation (28) states that $W$ must be chosen so that the marginal return from work must equal the marginal rate of substitution of consumption for labor. We assume that the government sets $s_{W}=\theta_{W}$ to offset the effect of the distortion associated with monopolistic competition in the labor market. Under this assumption the ratio $\frac{s_{W}}{\theta_{W}}$ equals one, so it does not appear in what follows, and the implied version of equation (28) states that the real wage must equal the marginal rate of substitution.

\subsection{Government}

The government budget constraint is

$$
\frac{M-M_{-1}+B^{g}-I_{-1} B_{-1}^{g}}{P}=G+\left(s_{P}-1\right) Y+\left(s_{W}-1\right) \frac{W}{P} L-T
$$


where $G$ is real government spending. We impose simple assumptions about the paths of government spending, interest payments, subsidy payments, and taxes under which we can study alternative monetary policy reaction functions. ${ }^{10}$. In particular, we assume that the government budget is balanced period by period and that real government spending is always zero, so the government budget constraint becomes ${ }^{11}$

$$
\frac{i_{-1} B_{-1}^{g}}{P}+\left(s_{P}-1\right) Y+\left(s_{W}-1\right) \frac{W}{P} L-T=0
$$

We assume that the government follows a monetary policy rule in the class

$$
I=\beta^{-1} P^{\lambda_{P}} Y^{\lambda_{Y}} Y^{* \lambda_{Y^{*}}} \bar{Y}^{\lambda_{\bar{Y}}} M^{\lambda_{M}} U^{\lambda_{U}} V^{\lambda_{V}} X^{\lambda_{X}} Z^{\lambda_{Z}}
$$

where $\bar{Y}$ is a target level of output. For rules in this class, either the price level or the money supply is the 'nominal anchor;' the sum of $\lambda_{P}$ and $\lambda_{M}$ must be non-zero in order for the price level to be determined with flexible wages and prices or oneperiod contracts for prices, wages, or both. We derive the optimal $\lambda_{j}$, the ones that maximize expected welfare. We also consider some alternative values of the $\lambda_{j}$.

\section{$3 \quad$ Flexible Wages and Prices}

We consider four versions of our model. To establish a benchmark, we begin by considering the version with flexible wages and prices.

\subsection{Solution}

In each version of the model six equations are used to determine the equilibrium values of the variables. With flexible wages and prices the forms of these six equations are

$$
\begin{aligned}
& Y=\frac{L^{\tilde{\alpha}} X}{\tilde{\alpha}}, \\
& P=\frac{L^{\alpha} W}{X},
\end{aligned}
$$

\footnotetext{
${ }^{10}$ Assumptions about the paths of government spending and taxes have implications for which monetary policies are feasible and for the effects of different feasible monetary policies. For an up to date discussion of the interaction between monetary and fiscal policy and citations of other recent contributions see Canzoneri, Cumby, and Diba (1998).

${ }^{11}$ We assume a monetary policy reaction function that implies that the expected rate of inflation, the solution for inflation in the model with flexible wages and prices when all shocks take on their mean values, is equal to zero. The analysis could be modified to allow for a nonzero expected rate of inflation. If the expected rate of rate of inflation were positive, the expected government deficit would have to be positive.
} 


$$
\begin{gathered}
\frac{\chi_{0} L^{\tilde{\chi}}}{W Z}=\frac{L}{Y^{\rho} P}, \\
\beta I \mathcal{E}\left(\frac{U_{+1}}{Y_{+1}^{\rho} P_{+1}}\right)=\frac{U}{Y^{\rho} P}, \\
I=\beta^{-1} P^{\lambda_{P}} Y^{\lambda_{Y}} Y^{* \lambda_{Y^{*}}} \bar{Y}^{\lambda_{\bar{Y}}} M^{\lambda_{M}} U^{\lambda_{U}} V^{\lambda_{V}} X^{\lambda_{X}} Z^{\lambda_{Z}}, \\
M=P C V
\end{gathered}
$$

where we have imposed the equilibrium conditions that $C=Y$ and $C_{+1}=Y_{+1}$ and where $\tilde{\alpha}=1-\alpha$ and $\tilde{\chi}=1+\chi$. With flexible wages and prices, both wages and prices are set after the shocks are known and the only expected magnitudes are in the demand equation.

The solutions for selected variables are shown in Table 1. Substituting the solutions for these variables into the equations of the model yields the solutions for the other variables. ${ }^{12}$

Substituting the production and price equations into the wage equation and solving yields the solution for $L$ in equation (T1.1) where $\tilde{\rho}=\rho-1$. To solve for the price level we use the method of undetermined coefficients. Suppose that $P$ takes the form given in equation (T1.2). We find $\Omega, \omega_{U}, \omega_{V}, \omega_{X}$, and $\omega_{Z}$ by beginning with the demand equation and eliminating $Y$ and $Y_{+1}$ using the solution for $Y^{*}$ implied by the solution for $L^{*}$ in equation (T1.1), eliminating $P$ using the conjectured solution in equation (T1.2), and eliminating $I$ using the rule equation to obtain

$$
\begin{aligned}
-\left(1+\lambda_{P}+\lambda_{M}\right)\left(\ln \Omega+\omega_{U} u+\omega_{V} v+\omega_{X} x+\omega_{Z} z\right) \\
\quad=\left(\lambda_{Y^{*}}+\lambda_{Y}+\lambda_{M}\right) \ln \left(\tilde{\alpha}^{-1} H^{\tilde{\alpha}}\right)+\lambda_{\bar{Y}} \bar{y}+\ln \mathcal{E}\left(Q_{1}\right)+\left(\lambda_{U}-1\right) u \\
\quad+\left(\lambda_{V}+\lambda_{M}\right) v+\left(\frac{\lambda_{X} D+\tilde{\chi}\left(\lambda_{Y^{*}}+\lambda_{Y}\right)+\tilde{\chi} \rho}{D}\right) x+\left(\frac{\lambda_{Z} D+\tilde{\alpha}\left(\lambda_{Y^{*}}+\lambda_{Y}+\lambda_{M}\right)+\tilde{\alpha} \rho}{D}\right) z
\end{aligned}
$$

where lower case letters represent logarithms, $D$ is defined in equation $(T 1.1)$, and

$$
Q_{1}=U_{+1} Y_{+1}^{-\rho} P_{+1}^{-1}=U_{+1}^{1-\omega_{U}} V_{+1}^{-\omega_{V}} Z_{+1}^{-\omega_{Z}-\frac{\rho \tilde{\alpha}}{D}} X_{+1}^{-\omega_{X}-\frac{\rho \tilde{\chi}}{D}}
$$

If equation (32) is to hold for all $U, V, X$, and $Z$, it must be that the $\omega_{j}$ and $\Omega$ take on the values given in equations (T1.4) through (T1.6). Substituting the solution for $L^{*}$ and the implied solution for $Y^{*}$ into the period utility function and considerable rearranging yield the solution for utility. So that we can simplify expressions by using logarithms, we express utility in terms of loss, $\mathbb{L}$, by defining

\footnotetext{
${ }^{12}$ The properties of log normal distributions used in this paper are summarized in Appendix A.
} 


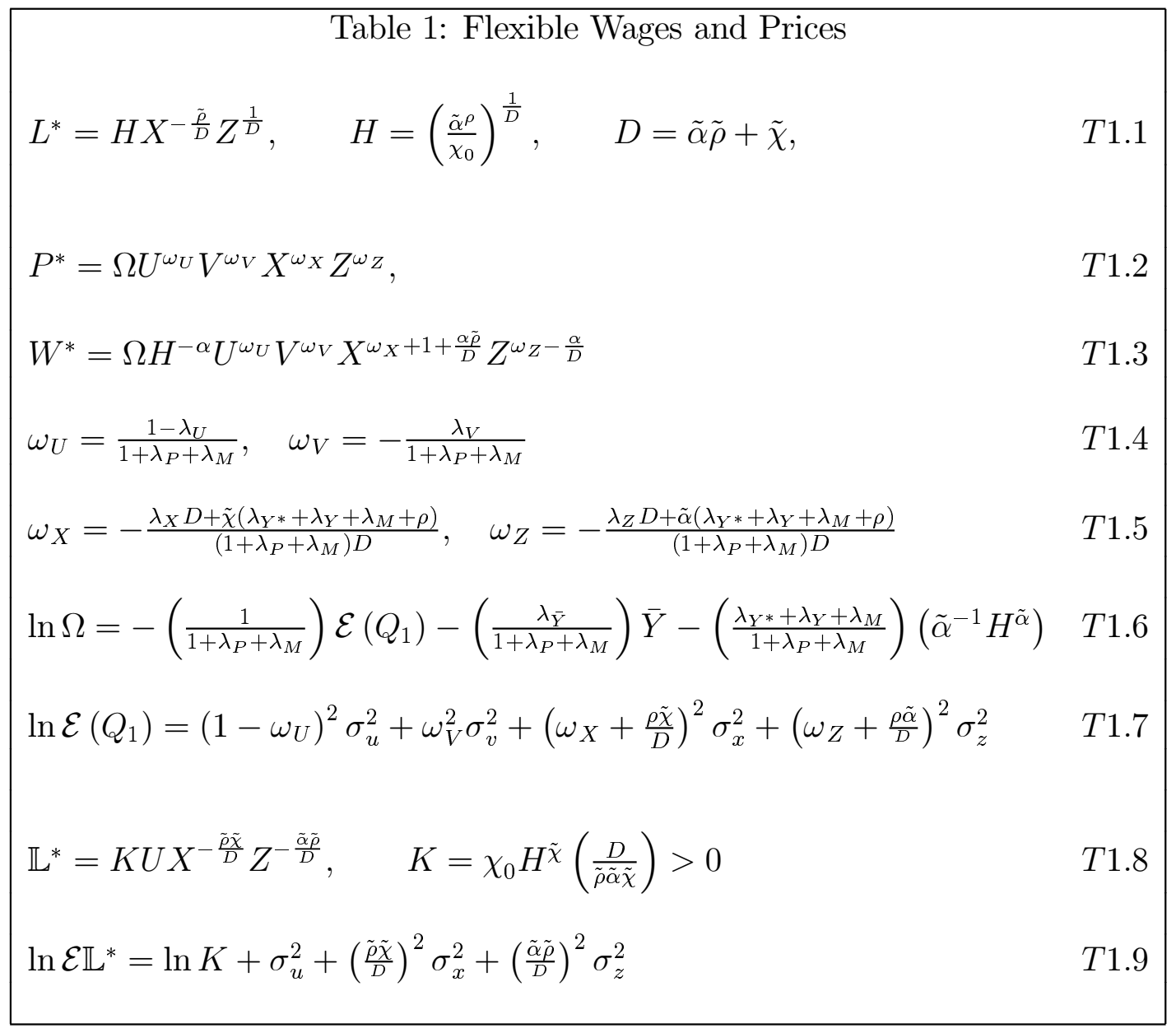

$$
\begin{aligned}
& \mathbb{L}=-\left(\frac{\mathcal{C}_{h, s}^{1-\rho}-1}{1-\rho}-\frac{\chi_{0} \mathrm{~L}_{h, s}^{1+\chi}}{Z_{s}(1+\chi)}+\frac{1}{1-\rho}\right) U_{s}>0
\end{aligned}
$$

The solution for loss is given in equation (T1.8). Taking expectations of equation (T1.8) yields the solution for expected loss in equation (T1.9).

\subsection{Discussion}

We are now prepared to discuss the effects of the shocks on the variables and utility. It is clear from Table 1 that our model passes the sunrise test. With flexible wages and prices, employment, $L$, and output, $Y$, the real variables that enter utility are independent of the money demand shock, $V$, and of the parameters of the monetary rule. Expected utility is independent of $\sigma_{v}^{2}$ and depends on $\sigma_{u}^{2}$ only because $U$ enters the utility function directly.

$L$ and $Y$ depend only on the productivity shock, $X$, and the labor supply shock, $Z$. The effects of a labor supply shock are easier to analyze than those of a productivity shock. The downward sloping marginal product of labor schedule, MPL, and the upward sloping marginal rate of substitution (of consumption for labor) schedule, 
$M R S$, implied by the price and wage equations, respectively are shown in the top panel of Figure 1 in logarithm space. An increase in $Z$ shifts the $M R S$ schedule down from $M R S_{0}$ to $M R S_{1}$. The equilibrium real wage must fall and equilibrium $l$ must rise from $l_{0}$ to $l_{1}$. The upward sloping production function schedule $P F$ is plotted in the bottom panel of Figure 1 in logarithm space. The increase in $Z$ does not affect the production function, so $y$ rises from $y_{0}$ to $y_{1}$ as $l$ rises from $l_{0}$ to $l_{1}$. An increase in $Z$ raises utility because it results in both an increase in the utility from consumption and a net reduction in the disutility of labor since we assume that $\tilde{\rho}>0$.

Under our assumptions, an increase in $X$ increases $y$ and lowers $l$. An increase in $X$ shifts both the $M P L$ and $M R S$ schedules up from $M P L_{0}$ to $M P L_{2}$ and from $M R S_{0}$ to $M R S_{2}$, respectively. Under our assumption that $\tilde{\rho}>0$, it shifts the $M R S$ schedule up by more. Therefore, the equilibrium real wage must rise and equilibrium $l$ must fall. An increase in $X$ also shifts the production function to the left from $P F_{0}$ to $P F_{2}$ and by more than it shifts the $M R S$ to the left because it takes less of a fall in $l$ to keep output constant than to keep households content with the same real wage. Thus, even though equilibrium $l$ falls, equilibrium $y$ rises. An increase in $X$ raises utility because it results in both an increase in the utility from consumption and a decrease in the disutility of labor.

$L$ and $Y$ do not depend on the goods demand shock, $U$, or the money demand shock, $V$. With flexible wages and prices, the model is recursive. The real variables, labor, output, and the real wage, are determined by the subsystem made up of the production, price, and wage equations. Given values of these variable, the nominal variables, the price level, the nominal interest rate, and the money supply, are determined by the subsystem made up of the demand, rule, and money equations. Neither $U$ nor $V$ enters the subsystem that determines the real variables. An increase in $U$ affects the utility of consumption and the disutility of labor in exactly the same way, so households have no incentive to change their decisions. Both $U$ and $V$ enter the subsystem that determines the nominal variables through the policy rule.

Increases in $\sigma_{u}^{2} \sigma_{x}^{2}, \sigma_{z}^{2}$, the variances of the logarithms of $U, X$, and $Z$, respectively, increase expected loss.

\section{Wage Contracts and Flexible Prices}

In this section, we consider the version with wage contracts and flexible prices.

\subsection{Solution}

In this version, the wage and price equations are

$$
\begin{gathered}
P=\frac{L^{\alpha} W}{X}, \\
\frac{1}{W} \mathcal{E}\left(\frac{\chi_{0} L^{\tilde{\chi}} U}{Z}\right)=\mathcal{E}\left(\frac{L U}{Y^{\rho} P}\right),
\end{gathered}
$$


The price equation is the same as in the case of perfectly flexible wages and prices, but the wage equation is different. With wage contracts, wages must be set one period in advance without knowledge of the current shocks, so the wage equation contains expectations.

As before, we solve the model using the method of undetermined coefficients. The solutions for selected variables are displayed in Table 2. The solutions for the other variables can be obtained using these solutions and the equations of the model. Suppose that solution for $L$ takes the form given in equation (T2.1). We find $\Xi$ by substituting the output and price equations into the wage equation and collecting terms to obtain

$$
\chi_{0} \mathcal{E}\left(\frac{L^{\tilde{\chi}} U}{Z}\right)=\tilde{\alpha}^{\rho} \mathcal{E}\left(\frac{U}{L^{\tilde{\alpha} \tilde{\rho}} X^{\tilde{\rho}}}\right) .
$$

Substituting in the conjectured form of the solution for $L$ in equation (T2.1)yields

$$
\begin{gathered}
\chi_{0} \Xi^{\tilde{\chi}} \mathcal{E} Q_{3}=\tilde{\alpha}^{\rho} \Xi^{-\tilde{\alpha} \tilde{\rho}} \mathcal{E} Q_{2}, \\
Q_{2}=U^{1-\xi_{U} \tilde{\alpha} \tilde{\rho}} V^{-\xi_{V} \tilde{\alpha} \tilde{\rho}} X^{-\left(\xi_{X} \tilde{\alpha}+1\right) \tilde{\rho}} Z^{-\xi_{Z} \tilde{\alpha} \tilde{\rho}}, \quad Q_{3}=U^{\xi_{U} \tilde{\chi}+1} V^{\xi_{V} \tilde{\chi}} X^{\xi_{X} \tilde{\chi}} Z^{\xi_{Z} \tilde{\chi}-1},
\end{gathered}
$$

Therefore, if equation (36) is to hold, $\Xi$ must take on the value in equation (T2.3).

We can find the $\xi_{j}$ and $W$ by substituting the rule equation into the demand equation and collecting terms to obtain

$$
U Y^{-\rho} P^{-1}=P^{\lambda_{P}} Y^{\lambda_{Y}} Y^{* \lambda_{Y^{*}}} \bar{Y}^{\lambda_{\bar{Y}}} M^{\lambda_{M}} U^{\lambda_{U}} V^{\lambda_{V}} X^{\lambda_{X}} Z^{\lambda_{Z}} \mathcal{E}\left(U_{+1} Y_{+1}^{-\rho} P_{+1}^{-1}\right)
$$

In a stationary rational expectations equilibrium with a levels reaction function $W_{+1}=W$. Imposing this restriction and eliminating $Y, P, M$, and $Y^{*}$ using the output, price, and money equations and the solution for $Y^{*}$ implied by the solution for $L^{*}$ in equation $(T 1.1)$, respectively, and collecting some terms yields

$$
\begin{gathered}
\left(\lambda_{M}+\tilde{\alpha}\left(\rho+\lambda_{Y}\right)+\alpha\left(1+\lambda_{P}\right)\right)\left(\ln \Xi+\xi_{U} u+\xi_{V} v+\xi_{X} x+\xi_{Z} z\right) \\
=-\left(\lambda_{Y}+\lambda_{M}\right) \ln \tilde{\alpha}^{-1}-\lambda_{Y^{*}} \ln \left(\tilde{\alpha}^{-1} H^{\tilde{\alpha}}\right)-\lambda_{\bar{Y}} \bar{y}+\ln \mathcal{E}\left(Q_{4}\right)-\left(\lambda_{P}+\lambda_{M}\right) w \\
+\left(1-\lambda_{U}\right) u-\left(\lambda_{V}+\lambda_{M}\right) v-\left(\frac{\left(\lambda_{X}+\tilde{\rho}-\lambda_{P}+\lambda_{Y}\right) D+\tilde{\chi} \lambda_{Y^{*}}}{D}\right) x-\left(\frac{\lambda_{Z} D+\tilde{\alpha} \lambda_{Y^{*}}}{D}\right) z \\
Q_{4}=U^{1-\xi_{U}(\tilde{\alpha} \rho+\alpha)} V^{-\xi_{V}(\tilde{\alpha} \rho+\alpha)} X^{-\left(\tilde{\rho}+\xi_{X}(\tilde{\alpha} \rho+\alpha)\right)} Z^{-\xi_{Z}(\tilde{\alpha} \rho+\alpha)}
\end{gathered}
$$

If equation (38) is to hold for all $U, V, X$, and $Z$, then the $\xi_{j}$ and $W$ must take on the values given in equations $(T 2.2)$ and $(T 2.8)$, respectively. 


\begin{tabular}{|lr|}
\hline \multicolumn{1}{|c}{ Table 2: Wage Contracts and Flexible Prices } & \\
$L=\Xi U^{\xi_{U}} V^{\xi_{V}} X^{\xi_{X}} Z^{\xi_{Z}}$, & $T 2.1$ \\
$\xi_{U}=\frac{1-\lambda_{U}}{\Gamma}, \xi_{V}=-\frac{\lambda_{V}+\lambda_{M}}{\Gamma}, \xi_{X}=-\frac{\lambda_{X}+\tilde{\rho}-\lambda_{P}+\lambda_{Y}}{\Gamma}-\frac{\tilde{\chi} \lambda_{Y^{*}}}{\Gamma D}, \xi_{Z}=-\frac{\lambda_{Z}}{\Gamma}-\frac{\tilde{\alpha} \lambda_{Y^{*}}}{\Gamma D}$ & $T 2.2$ \\
$\Xi=H\left(\frac{\mathcal{E} Q_{2}}{\mathcal{E} Q_{3}}\right)^{\frac{1}{D}}, \quad \Gamma=\lambda_{M}+\tilde{\alpha}\left(\rho+\lambda_{Y}\right)+\alpha\left(1+\lambda_{P}\right), \quad D=\tilde{\alpha} \tilde{\rho}+\tilde{\chi}$, & $T 2.3$ \\
$\ln \mathcal{E} Q_{2}=\left(1-\xi_{U} \tilde{\alpha} \tilde{\rho}\right)^{2} \sigma_{u}^{2}+\xi_{V}^{2} \tilde{\alpha}^{2} \tilde{\rho}^{2} \sigma_{v}^{2}+\left(\xi_{X} \tilde{\alpha}+1\right)^{2} \tilde{\rho}^{2} \sigma_{x}^{2}+\xi_{Z}^{2} \tilde{\alpha}^{2} \tilde{\rho}^{2} \sigma_{z}^{2}$ & $T 2.4$ \\
$\ln \mathcal{E} Q_{3}=\left(\xi_{U} \tilde{\chi}+1\right)^{2} \sigma_{u}^{2}+\xi_{V}^{2} \tilde{\chi}^{2} \sigma_{v}^{2}+\xi_{X}^{2} \tilde{\chi}^{2} \sigma_{x}^{2}+\left(\xi_{Z} \tilde{\chi}-1\right)^{2} \sigma_{z}^{2}$ & $T 2.5$ \\
$\ln \left(\frac{\mathcal{E} Q_{2}}{\mathcal{E} Q_{3}}\right)^{\frac{1}{D}}=\left(\xi_{U}^{2} \Lambda-2 \xi_{U}\right) \sigma_{u}^{2}+\xi_{V}^{2} \Lambda \sigma_{v}^{2}+\left(\frac{\xi_{X}^{2} D \Lambda+\left(2 \xi_{X} \tilde{\alpha}+1\right) \tilde{\rho}^{2}}{D}\right) \sigma_{x}^{2}+\left(\frac{\xi_{Z}^{2} D \Lambda+2 \xi_{Z} \tilde{\chi}-1}{D}\right) \sigma_{z}^{2}$ & $T 2.6$ \\
$\Lambda=\tilde{\alpha} \tilde{\rho}-\tilde{\chi}$ & $T 2.7$ \\
$W=\left(\Xi^{\Gamma} \tilde{\alpha}^{-\left(\lambda_{Y}+\lambda_{M}\right)}\left(\tilde{\alpha}^{-1} H^{\tilde{\alpha}}\right)^{\lambda_{Y *}} \bar{Y}^{\lambda_{\tilde{Y}}} \mathcal{E}\left(Q_{4}\right)\right)^{\frac{1}{\lambda_{P}+\lambda_{M}}}$ & $T 2.8$ \\
$\ln \mathcal{E}\left(Q_{4}\right)=\left(\left(\frac{1}{\tilde{\alpha} \rho+\alpha}-\xi_{U}\right)^{2} \sigma_{u}^{2}+\xi_{V}^{2} \sigma_{v}^{2}+\left(\xi_{X}+\frac{\tilde{\rho}}{\tilde{\alpha} \rho+\alpha}\right)^{2} \sigma_{x}^{2}+\xi_{Z}^{2} \sigma_{z}^{2}\right)(\tilde{\alpha} \rho+\alpha)^{2}$ & $T 2.9$ \\
\hline
\end{tabular}

\subsection{Expected Loss}

With wage contracts, the solutions for all the variables depend on the parameters of the monetary rule. In this subsection we derive the optimal rule with wage contracts and describe the effects of the shocks under that rule. Note that there is a one to one mapping from the parameters of the policy rule to the coefficients of the shocks in the solution for $L$. It is more convenient to determine the optimal shock coefficients for $L$ and then infer the optimal policy rule parameters.

The (logarithm of the) policymaker's expected loss is given by

$$
\begin{aligned}
\ln \mathcal{E} \mathbb{L} & =\ln K+\left(\xi_{U}^{2} \tilde{\alpha} \tilde{\rho} \tilde{\chi}+1\right) \sigma_{u}^{2}+\xi_{V}^{2} \tilde{\alpha} \tilde{\rho} \tilde{\chi} \sigma_{v}^{2} \\
& +\left(\left(\xi_{X}+\frac{\tilde{\rho}}{D}\right)^{2} \tilde{\alpha} \tilde{\rho} \tilde{\chi}+\left(\frac{\tilde{\rho} \tilde{\chi}}{D}\right)^{2}\right) \sigma_{x}^{2}+\left(\left(\xi_{Z}-\frac{1}{D}\right)^{2} \tilde{\alpha} \tilde{\rho} \tilde{\chi}+\left(\frac{\tilde{\alpha} \tilde{\rho}}{D}\right)^{2}\right) \sigma_{z}^{2}
\end{aligned}
$$

The derivation of this exact expression is actually simpler than the derivation of the standard approximation.

It is more convenient to work with the deviation of the policymaker's expected loss from Pareto optimal expected loss, $\Delta \ln \mathcal{E} \mathbb{L}=\ln \mathcal{E} \mathbb{L}-\ln \mathcal{E} \mathbb{L}^{*}$, where 


$$
\frac{\Delta \ln \mathcal{E} \mathbb{L}}{\tilde{\alpha} \tilde{\rho} \tilde{\chi}}=\xi_{U}^{2} \sigma_{u}^{2}+\xi_{V}^{2} \sigma_{v}^{2}+\left(\xi_{X}+\frac{\tilde{\rho}}{D}\right)^{2} \sigma_{x}^{2}+\left(\xi_{Z}-\frac{1}{D}\right)^{2} \sigma_{z}^{2}
$$

obtained by subtracting the expression for Pareto optimal expected loss in equation (T1.9) from equation (39).

\subsection{Optimal Policy}

It is clear from inspection that the values of the shock coefficients in the solution for labor which minimize (40) are

$$
\xi_{U}=0, \quad \xi_{V}=0, \quad \xi_{X}=-\frac{\tilde{\rho}}{D}, \quad \xi_{Z}=\frac{1}{D}
$$

and that if the shock coefficients take on these values expected loss with wage contracts is equal to the Pareto optimal level of expected loss.

In characterizing the optimal policy rule, we assume that the policymaker adjusts the nominal interest rate only in response to the price level and the shocks:

$$
\lambda_{U}, \lambda_{V}, \lambda_{X}, \lambda_{Z} \gtreqless 0, \quad \lambda_{P}>0, \quad \lambda_{M}=\lambda_{Y}=\lambda_{Y^{*}}=0
$$

and that $\lambda_{P}$ is an arbitrary positive number. The optimal rule coefficients implied by the optimal labor coefficients are obtained by equating the expressions for the shock coefficients in equation (T2.2) to the optimal values of these coefficients given in equation (41) and solving for the policy rule parameters. The results are

$$
\lambda_{U}=1, \lambda_{V}=0, \lambda_{X}=-\frac{\tilde{\rho} \chi}{\tilde{\alpha} \tilde{\rho}+\tilde{\chi}}+\left(\frac{\tilde{\rho}+\tilde{\chi}}{\tilde{\alpha} \tilde{\rho}+\tilde{\chi}}\right) \lambda_{P}, \lambda_{Z}=-\frac{\tilde{\alpha} \rho+\alpha}{\tilde{\alpha} \tilde{\rho}+\tilde{\chi}}-\left(\frac{\alpha}{\tilde{\alpha} \tilde{\rho}+\tilde{\chi}}\right) \lambda_{P}
$$

The model exhibits determinacy for any positive value of $\lambda_{P}$, so the value $\lambda_{P}$ can be chosen arbitrarily. Once a value of $\lambda_{P}$ is chosen, the values of the other policy rule parameters are determined. The policymaker should not move the interest rate in response to movements in $U$ or $V$. It should lower the interest rate if $Z$ rises no matter what the positive value of $\lambda_{P}$ because the marginal disutility of labor varies inversely with $Z$, so output and employment should be increased. Whether it should raise or lower the interest rate if $X$ rises depends on the value of $\lambda_{P}$.

An alternative way of finding the optimal rule is less direct but more elegant. If wages and prices are perfectly flexible and the policymaker follows the optimal rule for which the coefficients are given in equation (43), then for all shocks the economy is at the Pareto optimum, and the wage is unaffected. The wage result can be confirmed by substituting the expressions for the $\lambda_{i}$ in equation (43) into the solution for $W^{*}$ in equation (T1.3). The wage result implies that when the policymaker follows the optimal rule, the outcomes for all the variables including wages are the same no matter 
whether wages are preset in contracts. That is, the requirement that wages must remain constant is not a constraint that prevents attainment of the Pareto optimum. It follows that an alternative way of finding the optimal rule in the version with wage contracts and flexible prices without ever calculating the solution for that version is to find the rule that keeps wages constant in the version with flexible wages and prices. $^{13}$

\subsection{Output Gap Stabilization}

If the nominal interest rate responds only to the output gap, that is, only to deviations of output from its Pareto-optimal level, so that

$$
\lambda_{Y}=-\lambda_{Y^{*}}>0, \quad \lambda_{P}>0, \quad \lambda_{M}=\lambda_{U}=\lambda_{V}=\lambda_{X}=\lambda_{Z}=0
$$

the values of the shock coefficients in the solution for labor are

$$
\begin{gathered}
\xi_{U}=\frac{1}{\Gamma_{Y}}, \quad \xi_{V}=0, \quad \xi_{X}=-\frac{\tilde{\rho}-\lambda_{P}+\lambda_{Y}}{\Gamma_{Y}}+\frac{\tilde{\chi} \lambda_{Y}}{\Gamma_{Y} D}, \quad \xi_{Z}=\frac{\tilde{\alpha} \lambda_{Y}}{\Gamma_{Y} D} \\
\Gamma_{Y}=\tilde{\alpha}\left(\rho+\lambda_{Y}\right)+\alpha\left(1+\lambda_{P}\right)
\end{gathered}
$$

where the subscript on $\Gamma$ indicates the special case under consideration. In this case, for example, $\Gamma_{Y}$ is equal to $\Gamma$ with $\lambda_{M}=0$. Recall that there must always be a nominal anchor, so $\lambda_{P}>0$ in $\Gamma_{Y}$. Clearly if $\lambda_{Y}=-\lambda_{Y^{*}} \rightarrow \infty$, the values of the shock coefficients in the solution for labor are the Pareto-optimal equilibrium values given in equation (41). That is, complete stabilization of the output gap yields the same result as the optimal policy discussed in the preceding subsection. This result makes sense because, as stated above, loss can be written as a function of output and shocks and because we assume that the policymaker knows the shocks and, therefore, can calculate the Pareto-optimal value of output.

\subsection{Nominal Income Stabilization and Related Hybrid Rules}

If the nominal interest rate responds only to deviations of nominal income from a constant target value $\bar{Y}$, so that

$$
\lambda_{P}=\lambda_{Y}>0, \quad \lambda_{Y}=-\lambda_{\bar{Y}}, \quad \lambda_{Y^{*}}=\lambda_{M}=\lambda_{U}=\lambda_{V}=\lambda_{X}=\lambda_{Z}=0
$$

\footnotetext{
${ }^{13}$ Analogous logic applies in the case with price contracts and flexible wages. That is, the optimal rule with price contracts is the rule that keeps prices constant with completely flexible prices and wages. As we show in Appendix B, outcomes with price contracts and flexible wages are the same as the outcomes with wage and price contracts for all variables except the nominal wage. Therefore, the optimal rule with wage and price contracts is the same as the optimal rule with price contracts and flexible wages.
} 
then the expected loss deviation is

$$
\frac{\left.\Delta \ln \mathcal{E} \mathbb{L}\right|_{G} ^{P Y}}{\tilde{\alpha} \tilde{\rho} \tilde{\chi}}=\left(\frac{1}{\tilde{\alpha} \rho+\alpha+\lambda_{Y}}\right)^{2} \sigma_{u}^{2}+\left(\frac{-\tilde{\rho}}{\tilde{\alpha} \rho+\alpha+\lambda_{Y}}+\frac{\tilde{\rho}}{D}\right)^{2} \sigma_{x}^{2}+\left(\frac{1}{D}\right)^{2} \sigma_{z}^{2}
$$

where the superscript after the vertical bar indicates which variable is being stabilized and the subscript after the vertical bar can take on three values: $G$ for general, $C$ for complete stabilization, and $O$ for optimal stabilization.

Under complete nominal income stabilization $\left(\lambda_{P}=\lambda_{Y}>0, \quad \lambda_{Y}=-\lambda_{\bar{Y}} \rightarrow \infty\right)$, the expected loss deviation is

$$
\frac{\left.\Delta \ln \mathcal{E} \mathbb{L}\right|_{C} ^{P Y}}{\tilde{\alpha} \tilde{\rho} \tilde{\chi}}=\left(\frac{\tilde{\rho}}{D}\right)^{2} \sigma_{x}^{2}+\left(\frac{1}{D}\right)^{2} \sigma_{z}^{2}
$$

Note that the more inelastic is labor supply (the larger $\chi$ and, therefore, the larger is $D)$ the closer is complete nominal income stabilization to the fully optimal policy. ${ }^{14}$

The policy that is optimal within the class of nominal income stabilization policies is found by minimizing the expected loss deviation in equation (47) with respect to $\lambda_{Y}$. The first order condition for $\lambda_{Y}$ and the optimal $\lambda_{Y}$ and $\xi$ 's are

$$
\begin{gathered}
0=D \sigma_{u}^{2}+\tilde{\rho}^{2} \chi \sigma_{x}^{2}-\lambda_{Y} \tilde{\rho}^{2} \sigma_{x}^{2} \\
\lambda_{Y}=\frac{D \sigma_{u}^{2}+\tilde{\rho}^{2} \chi \sigma_{x}^{2}}{\tilde{\rho}^{2} \sigma_{x}^{2}} \\
\xi_{U}=\frac{\tilde{\rho}^{2} \sigma_{x}^{2}}{D\left(\tilde{\rho}^{2} \sigma_{x}^{2}+\sigma_{u}^{2}\right)}, \quad \xi_{V}=0, \quad \xi_{X}=-\frac{\tilde{\rho}^{3} \sigma_{x}^{2}}{D\left(\tilde{\rho}^{2} \sigma_{x}^{2}+\sigma_{u}^{2}\right)}, \quad \xi_{Z}=0 .
\end{gathered}
$$

Therefore, the expected loss from optimal stabilization of output is a positive fraction of the loss associated with the productivity shock under complete stabilization of output plus the irreducible loss associated with the labor supply shock:

$$
\frac{\left.\Delta \ln \mathcal{E} U\right|_{O} ^{P Y}}{\tilde{\alpha} \tilde{\rho} \tilde{\chi}}=\left(\frac{\sigma_{u}^{2}}{\tilde{\rho}^{2} \sigma_{x}^{2}+\sigma_{u}^{2}}\right)\left(\frac{\tilde{\rho}}{D}\right)^{2} \sigma_{x}^{2}+\left(\frac{1}{D}\right)^{2} \sigma_{z}^{2}
$$

The fraction rises from zero to one as the ratio $\frac{\sigma_{u}^{2}}{\sigma_{x}^{2}}$ increases from zero to infinity.

Welfare is higher than with optimal nominal income stabilization if the policymaker completely stabilizes a combination of the price level and output in which the weights on the two variables are not equal. ${ }^{15}$ In particular, if

\footnotetext{
${ }^{14}$ This result was obtained by Bean (1983).

${ }^{15}$ This result was obtained by Koenig (1996).
} 


$$
\frac{\lambda_{P}}{\lambda_{Y}}=\frac{\tilde{\chi}}{\tilde{\rho}+\tilde{\chi}}>0, \quad \lambda_{Y}=-\lambda_{\bar{Y}} \rightarrow \infty, \quad \lambda_{Y^{*}}=\lambda_{M}=\lambda_{U}=\lambda_{V}=\lambda_{X}=\lambda_{Z}=0
$$

then the expected loss deviation is

$$
\frac{\left.\Delta \ln \mathcal{E} \mathbb{L}\right|_{O} ^{P, Y}}{\tilde{\alpha} \tilde{\rho} \tilde{\chi}}=\left(\frac{1}{D}\right)^{2} \sigma_{z}^{2}
$$

The optimal hybrid policy can achieve the Pareto optimal outcomes for three of the four shocks. With only wage contracts, there are four disturbance coefficients in the solution for labor, $\xi_{U}, \xi_{V}, \xi_{X}$, and $\xi_{Z}$. When a combination of the price level and output are stabilized, $\xi_{V}$ and $\xi_{Z}$ are equal to zero no matter what the values of the rule coefficients, $\lambda_{P}$ and $\lambda_{Y}$. Zero is the optimal value for $\xi_{V}$, but not for $\xi_{Z}$, so there is some irreducible loss. The two remaining disturbance coefficients, $\xi_{U}$ and $\xi_{X}$, are independent functions of the rule coefficients, $\lambda_{P}$ and $\lambda_{Y}$, so they can be set at their optimal values by the appropriate choices of values for these coefficients. A hybrid rule can do nothing to offset labor supply shocks. The realization of the labor supply shock does not enter the solution for output and the price level because only the expectation of the labor supply equation is in the set of equations that determines the equilibrium values of these variables.

There is an alternative way of finding the optimal hybrid rule which is analogous to the alternative way of finding the fully optimal rule discussed in the subsection on optimal policy. The optimal hybrid rule in the version with wage contracts and flexible prices is the rule that would make the nominal wage invariant to demand, money, and productivity shocks $(U, V$, and $X)$ in the version with flexible wages and prices. The solution for the nominal wage with flexible wages and prices is given in equation (T1.3) and with a hybrid rule the nominal wage is invariant to $U, V$, and $X$ if and only if the $\lambda_{i}$ are set at the values given in equation (53).

\subsection{Price Level Stabilization}

If the nominal interest rate responds only to deviations of the price from a constant target value, so that

$$
\lambda_{P}>0, \quad \lambda_{Y}=\lambda_{Y^{*}}=\lambda_{M}=\lambda_{U}=\lambda_{V}=\lambda_{X}=\lambda_{Z}=0
$$

then the expected loss deviation is

$$
\begin{gathered}
\frac{\left.\Delta \ln \mathcal{E} \mathbb{L}\right|_{G} ^{P}}{\tilde{\alpha} \tilde{\rho} \tilde{\chi}}=\left(\frac{1}{\Gamma_{P}}\right)^{2} \sigma_{u}^{2}+\left(\frac{\lambda_{P}-\tilde{\rho}}{\Gamma_{P}}+\frac{\tilde{\rho}}{D}\right)^{2} \sigma_{x}^{2}+\left(\frac{1}{D}\right)^{2} \sigma_{z}^{2} \\
\Gamma_{P}=\tilde{\alpha} \rho+\alpha+\alpha \lambda_{P}
\end{gathered}
$$


Under complete price level stabilization, the expected loss deviation is

$$
\frac{\left.\Delta \ln \mathcal{E} \mathbb{L}\right|_{C} ^{P}}{\tilde{\alpha} \tilde{\rho} \tilde{\chi}}=\left(\frac{1}{\alpha}+\frac{\tilde{\rho}}{D}\right)^{2} \sigma_{x}^{2}+\left(\frac{1}{D}\right)^{2} \sigma_{z}^{2}=\left(\frac{\rho+\chi}{\alpha D}\right)^{2} \sigma_{x}^{2}+\left(\frac{1}{D}\right)^{2} \sigma_{z}^{2}
$$

For productivity shocks, under price level stabilization, employment and, therefore, output are more volatile than under the optimal policy. For labor supply shocks, employment and, therefore, output are less volatile than under the optimal policy.

The policy that is optimal within the class of price stabilization policies is found by minimizing the expected loss deviation in equation (56) with respect to $\lambda_{P}$. The first order condition for $\lambda_{P}$ and the optimal $\lambda_{P}$ and $\xi$ 's are

$$
\begin{gathered}
0=\alpha \sigma_{u}^{2}+\left(\left(\lambda_{P}-\tilde{\rho}\right)+\Gamma_{P}\left(\frac{\tilde{\rho}}{D}\right)\right)\left(\left(\lambda_{P}-\tilde{\rho}\right) \alpha-\Gamma_{P}\right) \sigma_{x}^{2} \\
\lambda_{P}=\frac{\alpha D \sigma_{u}^{2}+\tilde{\rho} \rho \chi \sigma_{x}^{2}}{\rho(\rho+\chi) \sigma_{x}^{2}} \\
\xi_{U}=\frac{\rho(\rho+\chi) \sigma_{x}^{2}}{\left(\rho^{2} \sigma_{x}^{2}+\alpha^{2} \sigma_{u}^{2}\right)}, \quad \xi_{V}=0, \quad \xi_{X}=\frac{\alpha D \sigma_{u}^{2}-\tilde{\rho} \rho^{2} \sigma_{x}^{2}}{D\left(\rho^{2} \sigma_{x}^{2}+\alpha^{2} \sigma_{u}^{2}\right)}, \quad \xi_{Z}=0 .
\end{gathered}
$$

Therefore, the expected loss from optimal stabilization of the price level is a positive fraction of the loss associated with the productivity shock under complete stabilization of the price level plus the irreducible loss associated with the labor supply shock:

$$
\frac{\left.\Delta \ln \mathcal{E} \mathbb{U}\right|_{O} ^{P}}{\tilde{\alpha} \tilde{\rho} \tilde{\chi}}=\left(\frac{\alpha^{2} \sigma_{u}^{2}}{\rho^{2} \sigma_{x}^{2}+\alpha^{2} \sigma_{u}^{2}}\right)\left(\frac{\rho+\chi}{\alpha D}\right)^{2} \sigma_{x}^{2}+\left(\frac{1}{D}\right)^{2} \sigma_{z}^{2}
$$

The fraction rises from zero to one as the ration $\frac{\sigma_{u}^{2}}{\sigma_{x}^{2}}$ increases from zero to infinity.

\subsection{Output Stabilization}

If the nominal interest rate responds only to deviations of the output from a constant target value, so that

$$
\lambda_{Y}=-\lambda_{\bar{Y}}>0, \quad \lambda_{P}>0, \quad \lambda_{Y^{*}}=\lambda_{M}=\lambda_{U}=\lambda_{V}=\lambda_{X}=\lambda_{Z}=0
$$

then the expected loss deviation is

$$
\begin{gathered}
\frac{\left.\Delta \ln \mathcal{E} \mathbb{L}\right|_{G} ^{Y}}{\tilde{\alpha} \tilde{\rho} \tilde{\chi}}=\left(\frac{1}{\Gamma_{Y}}\right)^{2} \sigma_{u}^{2}+\left(\frac{\lambda_{P}-\lambda_{Y}-\tilde{\rho}}{\Gamma_{Y}}+\frac{\tilde{\rho}}{D}\right)^{2} \sigma_{x}^{2}+\left(\frac{1}{D}\right)^{2} \sigma_{z}^{2} \\
\Gamma_{Y}=\tilde{\alpha}\left(\rho+\lambda_{Y}\right)+\alpha\left(1+\lambda_{P}\right)
\end{gathered}
$$


Under complete output stabilization $\left(\lambda_{Y}=-\lambda_{\bar{Y}} \rightarrow \infty, \quad \lambda_{P}>0\right)$, the expected loss deviation is

$$
\frac{\left.\ln \mathcal{E} \mathbb{L}\right|_{C} ^{Y}}{\tilde{\alpha} \tilde{\rho} \tilde{\chi}}=\left(\frac{\tilde{\chi}}{\tilde{\alpha} D}\right)^{2} \sigma_{x}^{2}+\left(\frac{1}{D}\right)^{2} \sigma_{z}^{2}
$$

The policy that is optimal within the class of real output stabilization policies is found by minimizing the expected loss deviation in equation (??) with respect to $\lambda_{Y}$. The first order condition for $\lambda_{Y}$ and the optimal $\lambda_{Y}$ and $\xi$ 's are

$$
\begin{gathered}
0=\tilde{\alpha} D \sigma_{u}^{2}+\left(1+\lambda_{P}\right)\left[(\rho+\chi)\left(1+\lambda_{P}\right)-\tilde{\chi}\left(\rho+\lambda_{Y}\right)\right] \sigma_{x}^{2} \\
\lambda_{Y}=-\rho+\frac{(\rho+\chi) \tilde{\lambda}_{P}}{\tilde{\chi}}+\frac{\tilde{\alpha} D}{\tilde{\chi} \tilde{\lambda}_{P}} \frac{\sigma_{u}^{2}}{\sigma_{x}^{2}} \\
\xi_{U}=\frac{\tilde{\chi} \tilde{\lambda}_{P} \sigma_{x}^{2}}{D\left(\tilde{\lambda}_{P}^{2} \sigma_{x}^{2}+\tilde{\alpha}^{2} \sigma_{u}^{2}\right)}, \quad \xi_{V}=0, \quad \xi_{X}=-\frac{\tilde{\rho} \tilde{\lambda}_{P} \sigma_{x}^{2}+\tilde{\alpha} D \sigma_{u}^{2}}{D\left(\tilde{\lambda}_{P}^{2} \sigma_{x}^{2}+\tilde{\alpha}^{2} \sigma_{u}^{2}\right)}, \quad \xi_{Z}=0
\end{gathered}
$$

where $\tilde{\lambda}_{P}=1+\lambda_{P}$. Therefore, the expected loss from optimal stabilization of output is a positive fraction of the loss associated with the productivity shock under complete stabilization of output plus the irreducible loss associated with the labor supply shock:

$$
\frac{\left.\Delta \ln \mathcal{E} \mathbb{U}\right|_{O} ^{Y}}{\tilde{\alpha} \tilde{\rho} \tilde{\chi}}=\left(\frac{\tilde{\alpha}^{2} \sigma_{u}^{2}}{\tilde{\lambda}_{P}^{2} \sigma_{x}^{2}+\tilde{\alpha}^{2} \sigma_{u}^{2}}\right)\left(\frac{\tilde{\chi}}{\tilde{\alpha} D}\right)^{2} \sigma_{x}^{2}+\left(\frac{1}{D}\right)^{2} \sigma_{z}^{2}
$$

The fraction increases from zero to one as the ration $\frac{\sigma_{u}^{2}}{\sigma_{x}^{2}}$ increases from zero to infinity.

\subsection{Money Supply Stabilization}

If the nominal interest rate responds only to deviations of the money supply from a constant target value, so that

$$
\lambda_{M}=-\lambda_{\bar{Y}}>0, \quad \lambda_{P}=\lambda_{Y}=\lambda_{Y^{*}}=\lambda_{U}=\lambda_{V}=\lambda_{X}=\lambda_{Z}=0
$$

then the expected loss deviation is

$$
\begin{gathered}
\frac{\left.\Delta \ln \mathcal{E} \mathbb{L}\right|_{G} ^{M}}{\tilde{\alpha} \tilde{\rho} \tilde{\chi}}=\left(\frac{1}{\Gamma_{M}}\right)^{2} \sigma_{u}^{2}+\left(\frac{\lambda_{M}}{\Gamma_{M}}\right)^{2} \sigma_{v}^{2}+\left(\frac{-\tilde{\rho}}{\Gamma_{M}}+\frac{\tilde{\rho}}{D}\right)^{2} \sigma_{x}^{2}+\left(\frac{1}{D}\right)^{2} \sigma_{z}^{2} \\
\Gamma_{M}=\lambda_{M}+\tilde{\alpha} \rho+\alpha
\end{gathered}
$$


Under complete money supply stabilization $\left(\lambda_{M}=-\lambda_{\bar{Y}} \rightarrow \infty\right)$, the expected loss deviation is

$$
\frac{\left.\Delta \ln \mathcal{E} \mathbb{L}\right|_{C} ^{M}}{\tilde{\alpha} \tilde{\rho} \tilde{\chi}}=\sigma_{v}^{2}+\left(\frac{\tilde{\rho}}{D}\right)^{2} \sigma_{x}^{2}+\left(\frac{1}{D}\right)^{2} \sigma_{z}^{2}
$$

The policy that is optimal within the class of money supply stabilization policies is found by minimizing the expected loss deviation in equation (70) with respect to $\lambda_{M}$. The first order condition for $\lambda_{M}$ and the optimal $\lambda_{M}$ and $\xi$ 's are

$$
\begin{gathered}
0=-D \sigma_{u}^{2}+\lambda_{M} D(\tilde{\alpha} \rho+\alpha) \sigma_{v}^{2}+\tilde{\rho}\left(-\tilde{\rho} D+\tilde{\rho} \Gamma_{M}\right) \sigma_{x}^{2} \\
\lambda_{M}=\frac{D \sigma_{u}^{2}+\tilde{\rho}^{2} \chi \sigma_{x}^{2}}{\tilde{\rho}^{2} \sigma_{x}^{2}+D(\tilde{\alpha} \rho+\alpha) \sigma_{v}^{2}} \\
\xi_{U}=\frac{J}{R}, \quad \xi_{V}=-\frac{D \sigma_{u}^{2}+\tilde{\rho}^{2} \chi \sigma_{x}^{2}}{R}, \quad \xi_{X}=-\frac{\tilde{\rho} J}{R}, \quad \xi_{Z}=0 . \\
J=\tilde{\rho}^{2} \sigma_{x}^{2}+D A \sigma_{v}^{2}, \quad R=D\left(\sigma_{u}^{2}+\tilde{\rho}^{2} \sigma_{x}^{2}+A^{2} \sigma_{v}^{2}\right), \quad A=\tilde{\alpha} \rho+\alpha
\end{gathered}
$$

The expected loss from optimal stabilization of the money supply is

$$
\begin{gathered}
\frac{\left.\Delta \ln \mathcal{E} \mathbb{U}\right|_{O} ^{M}}{\tilde{\alpha} \tilde{\rho} \tilde{\chi}}=\frac{\left(\sigma_{u}^{2}+\tilde{\rho}^{2} \sigma_{x}^{2}\right) \sigma_{u}^{2} \tilde{\rho}^{2} \sigma_{x}^{2}}{R^{2}}+\frac{\left(\tilde{\rho}^{2} \sigma_{x}^{2}+A^{2} \sigma_{v}^{2}\right) \tilde{\rho}^{2} \chi^{2} \sigma_{x}^{2} \sigma_{v}^{2}}{R^{2}} \\
+\frac{\left(\sigma_{u}^{2}+A^{2} \sigma_{v}^{2}\right) D^{2} \sigma_{u}^{2} \sigma_{v}^{2}}{R^{2}}+\frac{2 \tilde{\rho}^{2}\left(A^{2}+A \chi+\chi^{2}\right) \sigma_{u}^{2} \sigma_{v}^{2} \sigma_{x}^{2}}{R^{2}}+\left(\frac{1}{D}\right)^{2} \sigma_{z}^{2}
\end{gathered}
$$

Comparison of equation (75) with equation (52) confirms that if $\sigma_{u}^{2}, \sigma_{x}^{2}>0$, but $\sigma_{v}^{2}=0$, then the expected loss from optimal money supply stabilization is the same as the expected loss from optimal nominal income stabilization. However, if $\sigma_{x}^{2}, \sigma_{v}^{2}>0$, but $\sigma_{u}^{2}=0$ or $\sigma_{u}^{2}, \sigma_{v}^{2}>0$, but $\sigma_{x}^{2}=0$, expected loss from optimal money supply stabilization is larger than expected loss from optimal nominal income stabilization.

Although we have used our model to make clear the disadvantages of money supply stabilization, we cannot use it to evaluate claims about the advantages of this policy. In our model, all data become available simultaneously. However, in realworld economies money supply data become available more quickly than most, and it is sometimes claimed that money supply stabilization has an advantage because of this fact. In our model, the policymaker can achieve a desired value for any single variable. However, it is sometimes claimed that in real-world economies it is easier to achieve a desired value for the money supply than for some other variables. 


\section{$5 \quad$ Wage and Price Contracts}

In this section we consider the version with both wage and price contracts.

\subsection{Solution}

In this version, both the wage and price equations are different from the case of perfectly flexible wages and prices:

$$
\begin{gathered}
\mathcal{E}\left(\frac{U}{Y^{\tilde{\rho}}}\right)=\frac{W}{P} \mathcal{E}\left(\frac{L^{\alpha} U}{Y^{\tilde{\rho}} X}\right), \\
\mathcal{E}\left(\frac{\chi_{0} L^{\tilde{\chi}} U}{Z}\right)=\frac{W}{P} \mathcal{E}\left(\frac{L U}{Y^{\rho}}\right),
\end{gathered}
$$

Both wages and prices must be set one period in advance without knowledge of the current shocks so both the wage equation and the price equation contain expectations.

We solve the model using the method of undetermined coefficients. The solutions are displayed in Table 3. Suppose that the solution for $L$ has the form given in equation (T3.1). We find $\Psi$ by substituting the production equation into the price and wage equations, collecting terms, and dividing the price equation by the wage equation to eliminate $\frac{W}{P}$ to obtain

$$
\frac{\mathcal{E}\left(L^{-\tilde{\alpha} \tilde{\rho}} U X^{-\tilde{\rho}}\right)}{\chi_{0} \mathcal{E}\left(L^{\tilde{\chi}} U Z^{-1}\right)}=\frac{\mathcal{E}\left(L^{\alpha-\tilde{\alpha} \tilde{\rho}} U X^{-\rho}\right)}{\tilde{\alpha}^{\rho} \mathcal{E}\left(L^{\alpha-\tilde{\alpha} \tilde{\rho}} U X^{-\rho}\right)} .
$$

Substituting in the conjectured form of the solution for $L$ in equation (T3.1) and rearranging yields

$$
\begin{gathered}
\frac{\tilde{\alpha}^{\rho} \Psi^{-\tilde{\alpha} \tilde{\rho}} \mathcal{E}\left(Q_{5}\right)}{\chi_{0} \Psi \tilde{\chi} \mathcal{E}\left(Q_{6}\right)}=1 \\
Q_{5}=U^{1-\psi_{U} \tilde{\alpha} \tilde{\rho}} V^{-\psi_{V} \tilde{\alpha} \tilde{\rho}} X^{-\left(\psi_{X} \tilde{\alpha} \tilde{\rho}+\tilde{\rho}\right)} Z^{-\psi_{Z} \tilde{\alpha} \tilde{\rho}}, \quad Q_{6}=U^{\psi_{U} \tilde{\chi}+1} V^{\psi_{V} \tilde{\chi}} X^{\psi_{X} \tilde{\chi}} Z^{\psi_{Z} \tilde{\chi}-1}
\end{gathered}
$$

If equation (77) is to hold $\Psi$ must take on the value in equation (T3.3).

We find the $\psi_{j}, P$, and $W$ by substituting the rule equation into the demand equation to obtain

$$
Y^{-\rho} P^{-1} U=P^{\lambda_{P}} Y^{\lambda_{Y}} Y^{* \lambda_{Y} *} \bar{Y}^{\lambda_{\bar{Y}}} M^{\lambda_{M}} U^{\lambda_{U}} V^{\lambda_{V}} X^{\lambda_{X}} Z^{\lambda_{Z}} \mathcal{E}_{t}\left(Y_{+1}^{-\rho} P_{+1}^{-1} U_{+1}\right)
$$


Table 3: Wage and Price Contracts

$$
\begin{aligned}
& L=\Psi U^{\psi_{U}} V^{\psi_{V}} X^{\psi_{X}} Z^{\psi_{Z}} \\
& \psi_{U}=\frac{1-\lambda_{U}}{\digamma}, \psi_{V}=-\frac{\lambda_{V}+\lambda_{M}}{\digamma}, \psi_{X}=-\frac{\lambda_{X}+\rho+\lambda_{Y}+\lambda_{M}}{\digamma}-\frac{\tilde{\chi} \lambda_{Y^{*}}}{\digamma D}, \psi_{Z}=-\frac{\lambda_{Z}}{\digamma}-\frac{\tilde{\alpha} \lambda_{Y^{*}}}{\digamma D} \\
& \Psi=H\left(\frac{\mathcal{E} Q_{8}}{\mathcal{E} Q_{9}}\right)^{\frac{1}{D}}, \quad \digamma=\tilde{\alpha}\left(\rho+\lambda_{M}+\lambda_{Y}\right), \quad D=\tilde{\alpha} \tilde{\rho}+\tilde{\chi} \\
& \ln \mathcal{E} Q_{5}=\left(\psi_{U} \tilde{\alpha} \tilde{\rho}-1\right)^{2} \sigma_{u}^{2}+\psi_{V}^{2} \tilde{\alpha}^{2} \tilde{\rho}^{2} \sigma_{v}^{2}+\left(\psi_{X} \tilde{\alpha} \tilde{\rho}+\tilde{\rho}\right)^{2} \sigma_{x}^{2}+\psi_{Z}^{2} \tilde{\alpha}^{2} \tilde{\rho}^{2} \sigma_{z}^{2} \\
& \ln \mathcal{E} Q_{6}=\left(\psi_{U} \tilde{\chi}+1\right)^{2} \sigma_{u}^{2}+\psi_{V}^{2} \tilde{\chi}^{2} \sigma_{v}^{2}+\psi_{X}^{2} \tilde{\chi}^{2} \sigma_{x}^{2}+\left(\psi_{Z} \tilde{\chi}-1\right)^{2} \sigma_{z}^{2}, \\
& \ln \left(\frac{\mathcal{E} Q_{5}}{\mathcal{E} Q_{6}}\right)^{\frac{1}{D}}=\left(\psi_{U}^{2} \Lambda-2 \psi_{U}\right) \sigma_{u}^{2}+\psi_{V}^{2} \Lambda \sigma_{v}^{2}+\left(\frac{\psi_{X}^{2} \Lambda+\left(2 \psi_{X} \tilde{\alpha}+1\right) \tilde{\rho}^{2}}{D}\right) \sigma_{x}^{2}+\left(\frac{\psi_{Z}^{2} \Lambda+2 \psi_{Z} \tilde{\chi}-1}{D}\right) \sigma_{z}^{2} \\
& \Lambda=\tilde{\alpha} \tilde{\rho}-\tilde{\chi} \\
& P=\left(\Psi^{\digamma}\left(\tilde{\alpha}^{-1}\right)^{-\left(\lambda_{Y}+\lambda_{M}\right)}\left(\tilde{\alpha}^{-1} H^{\tilde{\alpha}}\right)^{\lambda_{Y^{*}}} \bar{Y}^{\lambda_{\bar{Y}}} \mathcal{E} Q_{7}\right)^{-\frac{1}{\lambda_{P}+\lambda_{M}}} \\
& \ln \mathcal{E} Q_{7}=\left(1-\psi_{U} \tilde{\alpha} \rho\right)^{2} \sigma_{u}^{2}+\psi_{V}^{2} \tilde{\alpha}^{2} \rho^{2} \sigma_{v}^{2}+\left(\psi_{X} \tilde{\alpha} \rho+\rho\right)^{2} \sigma_{x}^{2}+\psi_{Z}^{2} \tilde{\alpha}^{2} \rho^{2} \sigma_{z}^{2} \\
& W=P \Psi^{\tilde{\alpha} \rho+\tilde{\chi}+1}\left(\frac{\chi_{0}}{\tilde{\alpha}^{\rho}}\right)\left(\frac{\mathcal{E} Q_{5}}{\mathcal{E} Q_{8}}\right) \\
& \ln \mathcal{E} Q_{8}=\left(\left(\psi_{U}+\frac{1}{1-\tilde{\alpha} \rho}\right)^{2} \sigma_{u}^{2}+\psi_{V}^{2} \sigma_{v}^{2}+\left(\psi_{X}-\frac{\rho}{1-\tilde{\alpha} \rho}\right)^{2} \sigma_{x}^{2}+\psi_{Z}^{2} \sigma_{z}^{2}\right)(1-\tilde{\alpha} \rho)^{2}
\end{aligned}
$$

In a stationary rational expectations equilibrium with a levels reaction function $P_{+1}=$ $P$. Imposing this restriction and eliminating $Y, M$, and $Y^{*}$ using the production and money equations and the solution for $Y^{*}$ implied by the solution for $L^{*}$ in equation (T1.1), respectively, and collecting some terms yield

$$
\begin{gathered}
\tilde{\alpha}\left(\rho+\lambda_{M}+\lambda_{Y}\right)\left(\ln \Psi+\psi_{U} u+\psi_{V} v+\psi_{X} x+\psi_{Z} z\right) \\
=-\left(\lambda_{Y}+\lambda_{M}\right) \ln \tilde{\alpha}^{-1}-\lambda_{Y^{*}} \ln \left(\tilde{\alpha}^{-1} H^{\tilde{\alpha}}\right)-\lambda_{\bar{Y}} \bar{y}-\ln \mathcal{E}_{t}\left(Q_{7}\right)-\left(\lambda_{P}+\lambda_{M}\right) p \\
+\left(1-\lambda_{U}\right) u-\left(\lambda_{V}+\lambda_{M}\right) v-\left(\frac{\left(\lambda_{X}+\rho+\lambda_{Y}+\lambda_{M}\right) D+\tilde{\chi} \lambda_{Y^{*}}}{D}\right) x-\left(\frac{\lambda_{Z} D+\tilde{\alpha} \lambda_{Y^{*}}}{D}\right) z \\
Q_{7}=U^{1-\psi_{U} \tilde{\alpha} \rho} V^{-\psi_{V} \tilde{\alpha} \rho} X^{-\psi_{X} \tilde{\alpha} \rho-\rho} Z^{-\psi_{Z} \tilde{\alpha} \rho}
\end{gathered}
$$


If equation (79) is to hold for all $U, V, X$, and $Z$, it must be that the $\psi_{j}$ and $P$, respectively, must take on the values given in equations (T3.2) and (T3.8). Given the solution for $P$, the price equation can be used to obtain the solution for $W$ in equation $(T 3.10) \cdot{ }^{16}$

\subsection{Optimal Policy and Output Gap Stabilization}

In this subsection we discuss the optimal policy with wage and price contracts. As in the case of wage contracts and flexible prices, we state the policymaker's optimization problem in terms of the labor coefficients and then infer the optimal rule coefficients. It is clear from Tables 2 and 3 that the solutions for $L$ and, therefore, the solutions for $Y$ have exactly the same form with wage and price contracts as they do with wage contracts alone with $\psi_{j}, j=U, V, X, Z$ replacing $\xi_{j}, j=U, V, X, Z$ whereever they appear. It follows that the expressions for expected loss and, therefore, the optimal values of the shock coefficients in the solution for $L$ are the same with wage and price contracts as they are with wage contracts alone. That is,

$$
\psi_{U}=0, \quad \psi_{V}=0, \quad \psi_{X}=-\frac{\tilde{\rho}}{D}, \quad \psi_{Z}=\frac{1}{D}
$$

In characterizing the optimal policy rule, as before we assume that the policymaker responds only to the price level and the shocks:

$$
\lambda_{U}, \lambda_{V}, \lambda_{X}, \lambda_{Z} \gtreqless 0, \quad \lambda_{P}>0, \quad \lambda_{M}=\lambda_{Y}=\lambda_{Y^{*}}=0
$$

and that $\lambda_{P}$ is an arbitrary positive number. The optimal rule coefficients implied by the optimal labor coefficients are

$$
\lambda_{U}=1, \quad \lambda_{V}=0, \quad \lambda_{X}=-\frac{\rho \tilde{\chi}}{D}, \quad \lambda_{Z}=-\frac{\tilde{\alpha} \rho}{D}
$$

In contrast to the results for wage contracts alone, with wage and price contracts the optimal $\lambda_{j}, j=U, V, X, Z$ are independent of $\lambda_{P}$. The only role played by $\lambda_{P}$ is to guarantee determinacy, in particular, to insure that agents can calculate the expected future price level. The contract price for the current period is set before the shocks are drawn so there can be no movements in the current price level induced by the shocks and therefore nothing for the policymaker to respond to.

With wage and price contracts, just as with wage contracts alone, complete stabilization of the output gap yields the optimal outcome and for the same reason.

\subsection{Simple Policy Rules}

Given one-period wage and price contracts and the list of variables we have included in the policy rule, there are really only two simple rules to consider: output stabilization and money supply stabilization. Since prices are set before uncertainty is

\footnotetext{
${ }^{16}$ Of course, the solution for $W$ can also be obtained using the wage equation.
} 
resolved, the price level is always completely stabilized. As a consequence, stabilizing nominal income is the same thing as stabilizing output. Given the simple form of our money demand function, output stabilization and money supply stabilization have very similar implications. Stabilizing the money supply is the same thing as stabilizing output except that there is some increase in loss because shifts in money demand are not fully accommodated.

If the nominal interest rate responds only to deviations of output from the constant target value $\bar{Y}$, so that

$$
\lambda_{Y}=-\lambda_{\bar{Y}}>0, \quad \lambda_{P}>0, \quad \lambda_{Y^{*}}=\lambda_{M}=\lambda_{U}=\lambda_{V}=\lambda_{X}=\lambda_{Z}=0
$$

then the expected loss deviation is

$$
\frac{\left.\Delta \ln \mathcal{E} \mathbb{L}\right|_{G} ^{P Y}}{\tilde{\alpha} \tilde{\rho} \tilde{\chi}}=\left(\frac{1}{\tilde{\alpha}\left(\rho+\lambda_{Y}\right)}\right)^{2} \sigma_{u}^{2}+\left(-\frac{\tilde{\rho}+\lambda_{Y}}{\tilde{\alpha}\left(\rho+\lambda_{Y}\right)}+\frac{\tilde{\rho}}{D}\right)^{2} \sigma_{x}^{2}+\left(\frac{1}{D}\right)^{2} \sigma_{z}^{2}
$$

Under complete output stabilization $\left(\lambda_{Y}=-\lambda_{\bar{Y}} \rightarrow \infty, \lambda_{P}>0\right)$, the solutions for the $\psi_{j}$ are

$$
\psi_{U}=0, \quad \psi_{V}=0, \quad \psi_{X}=-\frac{1}{\tilde{\alpha}}, \quad \psi_{Z}=0 .
$$

and the expected loss deviation is

$$
\frac{\left.\Delta \ln \mathcal{E} \mathbb{L}\right|_{C} ^{P Y}}{\tilde{\alpha} \tilde{\rho} \tilde{\chi}}=\left(\frac{\tilde{\chi}}{\tilde{\alpha} D}\right)^{2} \sigma_{x}^{2}+\left(\frac{1}{D}\right)^{2} \sigma_{z}^{2}
$$

As is clear from a comparison of equations (86) and (48), if $\frac{\tilde{\chi}}{\tilde{\alpha}}>\tilde{\rho}$, that is, if the ratio of the elasticity of the disutility of labor to the labor elasticity of production exceeds the elasticity of the utility of consumption, complete nominal income stabilization increases loss more when there are price contracts.

The policy that is optimal within the class of output stabilization policies is found by minimizing the expected loss deviation in equation (84) with respect to $\lambda_{Y}$. The first order condition and the optimal $\lambda_{Y}$ and $\xi$ 's are

$$
\begin{gathered}
0=-\tilde{\alpha} D \sigma_{u}^{2}+\tilde{\rho}(\alpha+\chi) \sigma_{x}^{2}+\tilde{\alpha} \tilde{\chi} \sigma_{x}^{2} \lambda_{Y} \\
\lambda_{Y}=\frac{D \sigma_{u}^{2}-\tilde{\rho}(\alpha+\chi) \sigma_{x}^{2}}{\tilde{\chi} \sigma_{x}^{2}} \\
\xi_{U}=\frac{\tilde{\chi} \sigma_{x}^{2}}{\tilde{\alpha} D\left(\sigma_{u}^{2}+\sigma_{x}^{2}\right)}, \quad \xi_{V}=0, \quad \xi_{X}=-\frac{D \sigma_{u}^{2}+\tilde{\rho} \tilde{\alpha} \sigma_{x}^{2}}{\tilde{\alpha} D\left(\sigma_{u}^{2}+\sigma_{x}^{2}\right)}, \quad \xi_{Z}=0 .
\end{gathered}
$$


Therefore, the expected loss from optimal stabilization of output is a positive fraction of the loss associated with the productivity shock under complete stabilization of output plus the irreducible loss associated with the labor supply shock:

$$
\frac{\left.\Delta \ln \mathcal{E} U\right|_{O} ^{P Y}}{\tilde{\alpha} \tilde{\rho} \tilde{\chi}}=\left(\frac{\sigma_{u}^{2}}{\sigma_{u}^{2}+\sigma_{x}^{2}}\right)\left(\frac{\tilde{\chi}}{\tilde{\alpha} D}\right)^{2} \sigma_{x}^{2}+\left(\frac{1}{D}\right)^{2} \sigma_{z}^{2}
$$

The fraction rises from zero to one as the ration $\frac{\sigma_{u}^{2}}{\sigma_{x}^{2}}$ increases from zero to infinity.

\section{Conclusions}

In this paper we construct an optimizing-agent model with one-period nominal contracts which is simple enough that we can make exact utility calculations. We evaluate alternative monetary policy rules using as a criterion the utility function of the representative agent. We focus on the two cases of (1) wage contracts and flexible prices and (2) wage and price contracts because, as we show, the outcomes in the third case, price contracts and flexible wages, are the same as the outcomes in the case of wage and price contracts for all variables except the nominal wage.

The fully optimal rule under complete information can attain the Pareto-optimal equilibrium because we assume one-period nominal contracts. We contrast the performance of the fully optimal policy with both 'naive (complete stabilization)' and 'sophisticated (constrained optimal stabilization)' of one variable or a combination of two variables. The simple rules we consider can never achieve the Pareto-optimal outcome because they imply no response to labor supply shocks. However, if there are no labor supply shocks, in a few special cases, naive and optimal simple rules are as good as fully optimal rules. Of course, in general, they are not.

A number of our conclusions regarding simple rules depend critically on the relative importance of productivity disturbances. For example, with only wage contracts, the more important are productivity disturbances, the worse are all forms of nominal income targeting and the greater the difference between the naive and sophisticated versions. Another critical parameter is the elasticity of the disutility of labor (which, of course, is inversely related to the elasticity of labor supply). For example, if the elasticity of the disutility of labor is high with wage contracts alone naive nominal income targeting performs very well but with both wage and price contracts it performs very badly.

Just how much further it is worthwhile to push the analysis of one-period nominal contract models is an open question. In this paper, we reaffirm that such models are tractable, but we show that some of their results are quite special, for example the result that if there are price contracts the existence of wage contracts is of no consequence. In Henderson and Kim (1999a) we determine the effects of targeting money growth, inflation, and combinations of inflation and output on employment, output, and inflation. At a minimum, we plan to use the model of this paper to analyze the welfare implications of simple and optimal forms of these and related types of targeting. 


\section{Appendix A}

In this appendix we summarize the properties of log normal distributions that are used in this paper

Suppose that the variable $Q$ has a log normal distribution; that is, suppose that $q=\ln Q \sim N\left(\mu_{Q}, 2 \sigma_{Q}^{2}\right)$. Now $\ln Q^{k}=k q$ so $Q^{k}=e^{k q}$. It follows that the $E\left(Q^{k}\right)=$ $E\left(e^{k q}\right)=M(q, k)$ where $M(q, k)$ is the moment generating function for $q$ and is given by

$$
M(q, k)=\int_{-\infty}^{\infty} e^{k q}\left[\frac{1}{2 \sqrt{\pi} \sigma_{Q}} e^{-\frac{\left(q-\mu_{Q}\right)^{2}}{4 \sigma_{Q}^{2}}}\right] d q=e^{k \mu_{Q}+k^{2} \sigma_{Q}^{2}}
$$

that is

$$
E\left(Q^{k}\right)=e^{k \mu_{Q}+k^{2} \sigma_{Q}^{2}}
$$

Note that if $\mu_{Q}=0$, then $E(Q)=e^{\sigma_{Q}^{2}} \neq 1$ and $E\left(Q^{2}\right)=e^{4 \sigma_{Q}^{2}}$. However, if $E(Q)=1=e^{\mu_{Q}+\sigma_{Q}^{2}}$, then $0=\mu_{Q}+\sigma_{Q}^{2}$ so $\mu_{Q}=-\sigma_{Q}^{2}$ and $E\left(Q^{2}\right)=e^{2 \mu_{Q}+4 \sigma_{Q}^{2}}=e^{2 \sigma_{Q}^{2}}$. We have assumed that $\mu_{Q}=0$ in order to simplify our calculations. However, we can understand why others might prefer the alternative assumption.

Now suppose that the variables $U, V$, and $X$ are independently and log normally distributed; that is, suppose that $u=\ln U \sim N\left(\mu_{u}, 2 \sigma_{u}^{2}\right), v=\ln V \sim N\left(\mu_{v}, 2 \sigma_{v}^{2}\right)$, and $x=\ln X \sim N\left(\mu_{x}, 2 \sigma_{x}^{2}\right)$. It follows that

$$
E\left(U^{k_{U}} V^{k_{V}} X^{k_{X}}\right)=e^{k_{U} \mu_{u}+k_{U}^{2} \sigma_{u}^{2}+k_{V} \mu_{v}+k_{V}^{2} \sigma_{v}^{2}+k_{X} \mu_{x}+k_{X}^{2} \sigma_{x}^{2}}
$$




\section{Appendix B}

In this Appendix we show that the solutions with price contracts and flexible wages are the same as those with wage and price contracts for all variables except the nominal wages, as can be confirmed by comparing Table 4 with Table 3 . With price contracts and flexible wage the wage and price equations are

$$
\begin{aligned}
\mathcal{E}\left(\frac{U}{Y^{\tilde{\rho}}}\right) & =\frac{1}{P} \mathcal{E}\left(\frac{W L^{\alpha} U}{Y^{\tilde{\rho}} X}\right) \\
\frac{W}{P} & =\frac{\chi_{0} L^{\chi} Y^{\rho}}{Z}
\end{aligned}
$$

Suppose the solution for $L$ takes the form given in equation (T4.1). To find $\Phi$ we substitute the production and wage equations into the price equation, and collect terms:

$$
\chi_{0} \mathcal{E}\left(L^{\tilde{\chi}} U Z^{-1}\right)=\tilde{\alpha}^{\rho} \mathcal{E}\left(L^{-\tilde{\alpha} \tilde{\rho}} U X^{-\tilde{\rho}}\right)
$$

Substituting in the conjectured form for $L$ in equation (T4.1) in Table 4 yields

$$
\begin{gathered}
\chi_{0} \Phi^{\tilde{\chi}} \mathcal{E} Q_{6}=\tilde{\alpha}^{\rho} \Phi^{-\tilde{\alpha} \tilde{\rho}} \mathcal{E} Q_{5} \\
Q_{9}=U^{1-\phi_{U} \tilde{\alpha} \tilde{\rho}} V^{-\phi_{V} \tilde{\alpha} \tilde{\rho}} X^{-\left(\phi_{X} \tilde{\alpha} \tilde{\rho}+\tilde{\rho}\right)} Z^{-\phi_{Z} \tilde{\alpha} \tilde{\rho}}, \quad Q_{10}=U^{\phi_{U} \tilde{\chi}+1} V^{\phi_{V} \tilde{\chi}} X^{\phi_{x} \tilde{\chi}} Z^{\phi_{Z} \tilde{\chi}-1}
\end{gathered}
$$

If equation (B.2) is to hold $\Phi$ must take on the value given by equation (T4.3). Note that $Q_{9}, Q_{10}$, and $\Phi$ are identical to $Q_{2}, Q_{3}$, and $\Xi$ respectively except that $\xi_{j}$ is replaced by $\phi_{j}$ for $j=U, V, X$, and $Z$.

To find the $\phi_{j}$ and $P$ we substitute the rule equation into the demand equation:

$$
Y^{-\rho} P^{-1} U=P^{\lambda_{P}} Y^{\lambda_{Y}} Y^{* \lambda_{Y^{*}}} \bar{Y}^{\lambda_{\bar{Y}}} M^{\lambda_{M}} U^{\lambda_{U}} V^{\lambda_{V}} X^{\lambda_{X}} Z^{\lambda_{Z}}\left(Y_{+1}^{-\rho} P_{+1}^{-1} U_{+1}\right)
$$

Imposing the restriction that $P_{+1}=P$ and eliminating $Y, W, M$, and $Y^{*}$ using the production, wage, and money equations, and the solution for $Y^{*}$ implied by the solution for $L^{*}$ in equation $(T 1.1)$, respectively, and collecting terms yield

$$
\begin{aligned}
& \tilde{\alpha}\left(\rho+\lambda_{M}+\lambda_{Y}\right)\left(\ln \Phi+\phi_{U} u+\phi_{V} v+\phi_{X} x+\phi_{Z} z\right)=-\left(\lambda_{Y}+\lambda_{M}\right) \ln \left(\tilde{\alpha}^{-1}\right) \\
& -\lambda_{Y^{*}} \ln \left(\tilde{\alpha}^{-1} H^{\tilde{\alpha}}\right)-\lambda_{\bar{Y}} \bar{y}-\ln \mathcal{E}\left(Q_{11}\right)-\left(\lambda_{P}+\lambda_{M}\right) p \\
& +\left(1-\lambda_{U}\right) u-\left(\lambda_{V}+\lambda_{M}\right) v-\left(\frac{\left(\lambda_{X}+\rho+\lambda_{Y}+\lambda_{M}\right) D+\tilde{\chi} \lambda_{Y^{*}}}{D}\right) x-\left(\frac{\lambda_{Z} D+\tilde{\alpha} \lambda_{Y^{*}}}{D}\right) z
\end{aligned}
$$




\begin{tabular}{|lc|}
\hline \multicolumn{1}{|c}{ Table 4: Price Contracts and Flexible Wages } & \\
$L=\Phi U^{\phi_{U}} V^{\phi_{V}} X^{\phi_{X}} Z^{\phi_{Z}}$ & $T 4.1$ \\
$\phi_{U}=\frac{1-\lambda_{U}}{\digamma}, \phi_{V}=-\frac{\lambda_{V}+\lambda_{M}}{\digamma}, \phi_{X}=-\frac{\lambda_{X}+\rho+\lambda_{Y}+\lambda_{M}}{\digamma}-\frac{\tilde{\chi} \lambda_{Y^{*}}}{\digamma D}, \phi_{Z}=-\frac{\lambda_{Z}}{\digamma}-\frac{\tilde{\alpha} \lambda_{Y^{*}}}{\digamma D}$ & $T 4.2$ \\
$\Phi=H\left(\frac{\mathcal{E} Q_{9}}{\mathcal{E} Q_{10}}\right)^{\frac{1}{D}}, \quad \digamma=\tilde{\alpha}\left(\rho+\lambda_{M}+\lambda_{Y}\right), \quad D=\tilde{\alpha} \tilde{\rho}+\tilde{\chi}$ & $T 4.3$ \\
$\ln \mathcal{E} Q_{9}=\left(\phi_{U} \tilde{\alpha} \tilde{\rho}-1\right)^{2} \sigma_{u}^{2}+\phi_{V}^{2} \tilde{\alpha}^{2} \tilde{\rho}^{2} \sigma_{v}^{2}+\left(\phi_{X} \tilde{\alpha} \tilde{\rho}+\tilde{\rho}\right)^{2} \sigma_{x}^{2}+\phi_{Z}^{2} \tilde{\alpha}^{2} \tilde{\rho}^{2} \sigma_{z}^{2}$ & $T 4.4$ \\
$\ln \mathcal{E} Q_{10}=\left(\phi_{U} \tilde{\chi}+1\right)^{2} \sigma_{u}^{2}+\phi_{V}^{2} \tilde{\chi}^{2} \sigma_{v}^{2}+\phi_{X}^{2} \tilde{\chi}^{2} \sigma_{x}^{2}+\left(\phi_{Z} \tilde{\chi}-1\right)^{2} \sigma_{z}^{2}$, & $T 4.5$ \\
$\ln \left(\frac{\mathcal{E} Q_{9}}{\mathcal{E} Q_{10}}\right)^{\frac{1}{D}}=\left(\phi_{U}^{2} \Lambda-2 \phi_{U}\right) \sigma_{u}^{2}+\phi_{V}^{2} \Lambda \sigma_{v}^{2}+\left(\frac{\phi_{X}^{2} \Lambda+\left(2 \phi_{X} \tilde{\alpha}+1\right) \tilde{\rho}^{2}}{D}\right) \sigma_{x}^{2}+\left(\frac{\phi_{Z}^{2} \Lambda+2 \phi_{Z} \tilde{\chi}-1}{D}\right) \sigma_{z}^{2}$ & $T 4.6$ \\
$\Lambda=\tilde{\alpha} \tilde{\rho}-\tilde{\chi}$ & $T 4.7$ \\
$P=\left(\Phi^{\digamma}\left(\tilde{\alpha}^{-1}\right)^{-\left(\lambda_{Y}+\lambda_{M}\right)}\left(\tilde{\alpha}^{-1} H^{\tilde{\alpha}}\right)^{\lambda_{Y^{*}}} \bar{Y}^{\lambda_{\tilde{Y}}} \mathcal{E} Q_{7}\right)^{-\frac{1}{\lambda_{P}+\lambda_{M}}}$ & $T 4.8$ \\
$\ln \mathcal{E} Q_{11}=\left(\phi_{U} \tilde{\alpha} \rho-1\right)^{2} \sigma_{u}^{2}+\phi_{V}^{2} \tilde{\alpha}^{2} \rho^{2} \sigma_{v}^{2}+\left(\phi_{X} \tilde{\alpha} \rho+\rho\right)^{2} \sigma_{x}^{2}+\phi_{Z}^{2} \tilde{\alpha}^{2} \rho^{2} \sigma_{z}^{2}$ & $T 4.9$ \\
\hline
\end{tabular}

where $\ln \mathcal{E}\left(Q_{11}\right)$ is given by equation (T4.10). If equation (B.4) is to hold for all $U, V, X$, and $Z$, the $\phi_{j}$ and $P$ must take on the values given in equations (T4.2) and (T4.8), respectively. The solution for $W$ is found by substituting the solutions for $L$ and $P$ given by equations (T4.1) and (T4.8), respectively, and the solution for $Y$ implied by the solution for $L$ in equation (T4.1) into the wage equation (T4.10). 


\section{References}

Bean, C. (1983) "Targeting Nominal Income: An Appraisal", The Economic Journal, 93, 806-19.

Canzoneri, M. B., R. E. Cumby, and B. T. Diba (1998) "Is the Price Level Determined by the Needs of Fiscal Solvency?", processed, Georgetown University.

Corsetti, G., and P. Pesenti (1998) "Welfare and Macroeconomic Interdependence", processed, Yale University.

Devereux, M. B., and C. Engel (1998) "Fixed vs. Floating Exchange Rates: How Price Setting Affects the Optimal Choice of Exchange-Rate Regime", NBER Working Paper 6867, National Bureau of Economic Research.

Engel, C. (1999a) "On the Foreign Exchange Risk Premium in Sticky-Price General Equilibrium Models", International Tax and Public Finance, (forthcoming).

Engel, C. (1999b) "On the Foreign Exchange Risk Premium in Sticky-Price General Equilibrium Models", in P. Isard, A. Razin, and A. Rose (eds.) International Finance in Turmoil: Essays in Honor of Robert P. Flood, International Monetary Fund and Kluwer Academic Publishers, Washington and Boston, (forthcoming).

Erceg, C. J., D. W. Henderson, and A. T. Levin (1999) "Optimal Monetary Policy with Staggered Wage and Price Contracts", processed, Federal Reserve Board.

Goodfriend, M., and R. King (1997) "The New Neoclassical Synthesis and the Role of Monetary Policy", in NBER Macroeconomics Annual 1997, MIT Press, Cambridge, 233-283.

Henderson, D. W., and J. Kim (1999a) "The Choice of a Monetary Policy Reaction Function in a Simple Optimizing Model", in A. Leijonhufvud (ed.) Monetary Theory as a Basis for Monetary Policy, Macmillan, London, (forthcoming).

Henderson, D. W., and J. Kim (1999b) "Exact Utilities under Alternative Monetary Rules in a Simple Macro Model with Optimizing Agents", International Tax and Public Finance, (forthcoming).

Henderson, D. W., and J. Kim (1999c) "Exact Utilities under Alternative Monetary Rules in a Simple Macro Model with Optimizing Agents", in P. Isard, A. Razin, and A. Rose (eds.) International Finance in Turmoil: Essays in Honor of Robert P. Flood, International Monetary Fund and Kluwer Academic Publishers, Washington and Boston, (forthcoming).

Ireland, P. N. (1997) "A Small Structural, Quarterly Model for Monetary Policy Evaluation", in Carnegie-Rochester Series on Public Policy, Volume 47, 83-108.

Kim, J. (1997) "Three Sources of Increasing Returns to Scale", Finance and Economics Discussion Series 1997-18, Federal Reserve Board. 
Kim, J. (1998) "Specifications of Investment Adjustment Costs in a Simple Dynamic Model", Finance and Economics Discussion Series 1998-39, Federal Reserve Board.

Kim, J., and S. H. Kim (1999) "Inaccuracy of Loglinear Approximation in Welfare Calculation: Case of International Risk Sharing", processed, University of Virginia.

Koenig, E. F. (1996) "Targeting Nominal Income: A Closer Look", Economics Letters, $51,89-93$.

Obstfeld, M., and K. Rogoff (1998) "Risk and Exchange Rates", NBER Working Paper 6694, National Bureau of Economic Research.

Rotemberg, J. J., and M. Woodford (1998) "An Optimization-Based Econometric Framework for the Evaluation of Monetary Policy", in NBER Macroeconomics Annual 1997, MIT Press, Cambridge, 297-346.

Taylor, J. B. (ed.) (1999) Monetary Policy Rules, The University of Chicago Press, Chicago (forthcoming). 


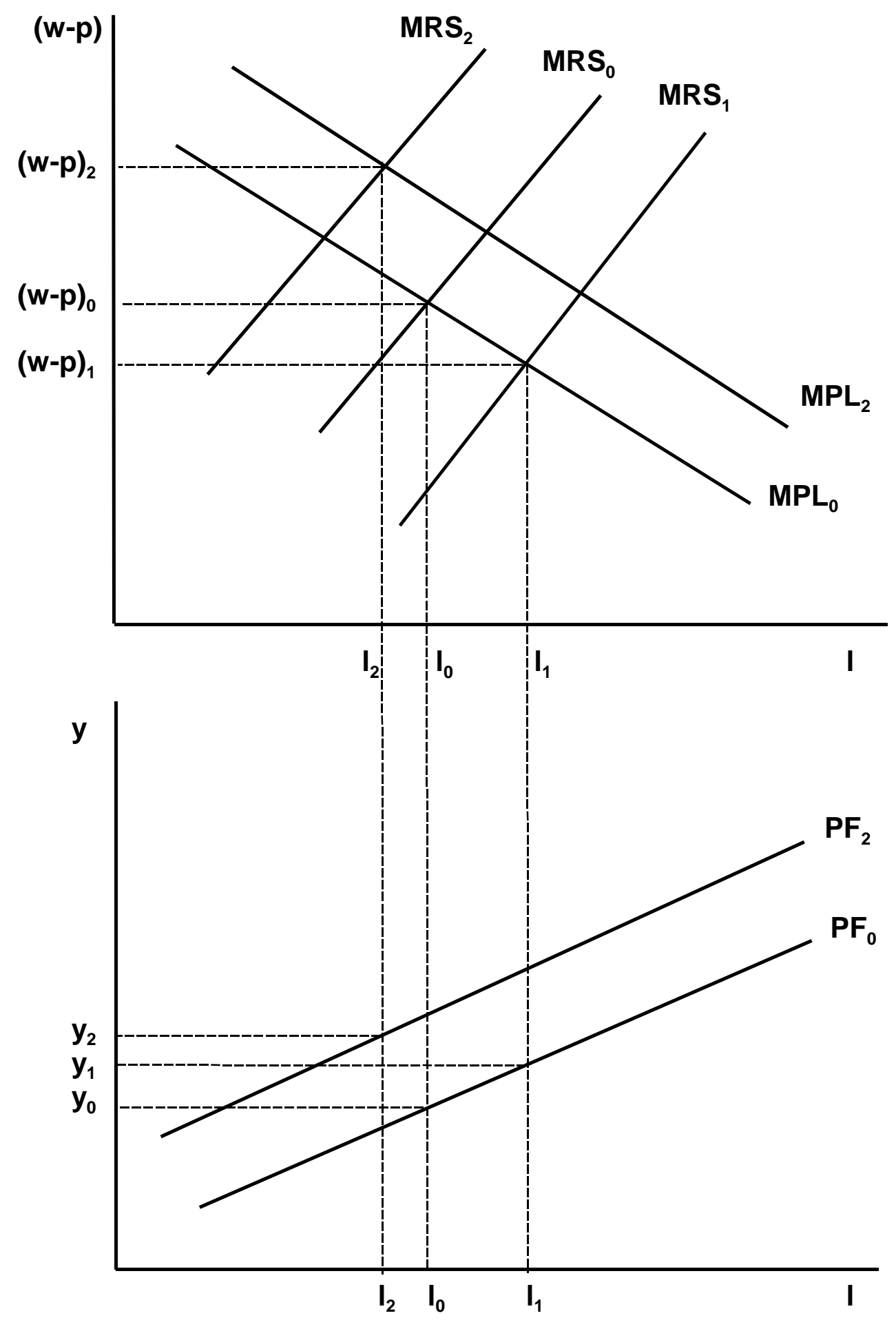

Figure 1. Flexible Wages and Prices 